This item was submitted to Loughborough's Research Repository by the author.

Items in Figshare are protected by copyright, with all rights reserved, unless otherwise indicated.

\title{
Study of an effusion-cooled plate with high level of upstream fluctuation
}

PLEASE CITE THE PUBLISHED VERSION

https://doi.org/10.1016/j.applthermaleng.2020.116126

PUBLISHER

Elsevier

VERSION

AM (Accepted Manuscript)

PUBLISHER STATEMENT

This paper was accepted for publication in the journal Applied Thermal Engineering and the definitive published version is available at https://doi.org/10.1016/j.applthermaleng.2020.116126

\section{LICENCE}

CC BY-NC-ND 4.0

\section{REPOSITORY RECORD}

Chen, Xiaosheng, Jason Krawciw, Hao Xia, Paul Denman, Clare Bonham, and Jon Carrotte. 2020. "Study of an Effusion-cooled Plate with High Level of Upstream Fluctuation". Loughborough University.

https://hdl.handle.net/2134/13095758.v1. 


\title{
Study of an effusion-cooled plate with high level of upstream fluctuation
}

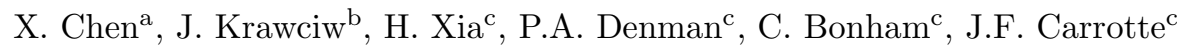 \\ ${ }^{a}$ Department of Engineering Science, The University of Oxford, Oxford, OX1 3PJ, UK \\ ${ }^{b}$ Rolls-Royce plc., PO Box 31, Derby, DE24 8BJ, UK \\ ${ }^{c}$ Department of Aeronautical and Automotive Engineering, Loughborough Univresity, Loughbrough, LE11 3TU, UK
}

\begin{abstract}
The flow field and surface adiabatic coolant-film effectiveness (ACE) distribution of a combustor representative effusion cooling array with cylindrical cooling holes has been studied both experimentally and numerically. Both studies focus on the influence of inflow turbulence, especially the high inflow turbulence which is always present in the combustor environment but rarely studied in the literature. A fluctuating inflow at roughly $20 \%$ intensity level is generated in the wind tunnel, and distributions of ACE measured for blowing ratios (BR) between 1.8 and 4.1. For comparison, ACE distributions are also measured at a low inflow turbulence intensity of $5 \%$. For further investigation on the mechanism of inflow turbulence effects, hybrid large eddy simulations (LES) are carried out at a BR of around 1.8 under both low and high inflow turbulence intensities. The fluctuating inflow is generated using the Synthetic Eddy Method (SEM) with similar turbulence intensity. The predicted surface ACE distributions of the 2 cases are compared with the measurements. More detailed studies of the flow field are carried out based on the numerical results. The effects of inflow fluctuation levels are studied by comparing various flow statistics between the low and high fluctuation cases. The formation of the coolant film is also studied based on the development of the coolant film thickness. The interaction between the upstream and downstream coolant jets is investigated by visualising the coolant jet centre trajectory, as well as analyzing the turbulence structures, spectra and coherence at selected positions. These analysis clearly show that the highly fluctuating inflow results in an enhanced mixing of the coolant and mainstream. In the high turbulence intensity case, this leads to wider span-wise and shorter stream-wise film coverage over the first few rows of the array. These effects diminish as soon as a thick coolant film is formed in the downstream, especially at high BR conditions.
\end{abstract}

Keywords: hybrid LES, Pressure Sensitive Paint, full-coverage effusion cooling, upstream fluctuation, adiabatic coolant-film effectiveness

\section{Introduction}

Modern gas turbine engines are operating at very high temperatures to achieve maximum possible thermal efficiency. However, this leads to the combustor chamber operating at an extremely hostile environment: incredibly high temperature combustion and highly unsteady mixing flow. The hot gas 5 after the combustion is well above the melting point of the combustor liner material. Various techniques have been developed to protect the liner from the extremely hot gas. These include thermal barrier coatings (TBC), where a ceramic material is placed between the hot gas and the metal surface, and 
the impingement cooling, where relatively cooler air flow is extracted from the last compressor stage and impinged on the hot spot of the metal through the channel inside the component, as well as the film cooling, where a film of low temperature air is formed on the liner surface isolating the metal from the hot gas by injecting the coolant through an array of small holes. Modern cooling designs make use of all these cooling techniques to protect the metal components and extend their service life as much as possible. However, the coolant air used by the impingement cooling and film cooling is taken from the compressor of the engine, and hence the available power produced by the combustor is reduced. Jet engine manufacturers are always looking for ways to increase the percentage of air for combustion, which results in less available air for cooling. As a consequence, jet engine manufacturers are concerned with the development of effusion cooling designs that reduce the coolant flow rate by improving cooling effectiveness. However, the mixing flow inside the combustor is highly unsteady with swirling jets and small vortices, thus making the coolant film extremely unstable and difficult to predict. A good understanding of the fundamental effusion cooling mechanism is therefore critical for the development of these design methods and significant number of studies have been carried out over the past decades both experimentally and numerically.

Experiments to determine adiabatic coolant-film effectiveness (ACE) generally involve measuring spatially resolved surface temperature distributions using techniques such as thermochromic liquid crystal 1, 2] and infra-red (IR) Thermography [3, 4]. The measurement surface is usually manufactured from a material with an extremely low thermal conductivity in order to satisfy the adiabatic condition. However, the impact of thermal conductivity can only be reduced, not eliminated entirely. Therefore, the measured temperatures must be corrected using approaches such as FE modelling or the implementation of the linear superposition principle [5].

To eliminate the requirement for an adiabatic measurement surface, the present study makes use of a pressure sensitive paint (PSP) technique and the analogy between heat and mass transfer [6]. PSP is an optical sensor that is sensitive to the partial pressure of oxygen. It can be used to obtain spatially resolved measurements of surface pressure when oxygen concentration is constant or oxygen concentration when the pressure field is known. In recent decades, the PSP technique has been applied to the measurement 35 of the static pressure field over aircraft bodies 7] and turbomachinery components [8, 9]. It has also been employed to measure the ACE over film-cooled turbine blades and nozzle guide vanes [10, 11]. In this latter application an oxygen-free coolant gas is used, which allows the ACE to be inferred from the local oxygen concentration provided the surface pressure distribution is known. More recently, the PSP technique is adopted to help study various different parameters in effusion/film cooling, such as BR 12] 40 and cooling hole arrangement 13$]$.

Amongst the published research on effusion cooling, very little is found on ACE distributions with inflow turbulence. Some experiments [14-16] found that a 3 to $8 \%$ inflow turbulence intensity resulted in a $15 \%$ reduction in ACE. Studies are lacking on the effects of higher levels of turbulence intensity, such as $20 \%$, which are typical in real combustor environment. Ziefle and Kleiser [17] carried out an LES study with turbulent mainstream. It was found that the turbulent inflow enhances the spreading of the coolant and considerable differences were found in the mixing region compared to those with a 
steady mainstream. However, the inflow in this study is involves a turbulent boundary layer rather than a fully disturbed inflow representing the combustor environment. Wurm et al.[18] made measurements of an effusion cooled plate at realistic combustor conditions with a swirling inflow at 2 liner pressure drops using the IR and PIV technology. However, the analysis to link the turbulent flow structures to the ACE performance was limited. Similar research was done by Andreini et al. [19] using the PSP technique. Although the ACE distribution was studied more carefully, the understanding of the relationship between the turbulent flow structures and the coolant film formation was still limited.

In this paper, experiments are carried out using the PSP technique to evaluate the coolant film performance of a combustor representative effusion array under high and low levels of upstream fluctuations. The aim of the experiment is to investigate the performance of the effusion cooling arrangement at scaled combustor conditions including the highly unstable upstream flow conditions. However, the limited data available from experiments means that the relationship between flow features and coolant film performance cannot be understood. Hence, hybrid LES-RANS simulations are conducted at a selected blowing ratio, with synthetically generated inflow fluctuations. The numerical simulations aim to investigate the detailed interaction between the coolant jets and the mainstream, as well as the effects on the surface ACE distribution.

A brief introduction to the structure of this paper is presented. The experimental facilities, configurations of the cases and the measured results and analysis are described in Section 2. The numerical methodology and computational domain are explained in Section 3, with a brief description of a separate grid resolution study. Section 4 presents the numerical results and analysis. This section starts with comparison and analysis of ACE distribution between measurements and simulations, followed by detailed investigations on coolant film formation, flow structures and spectral analysis. A brief summary of this study is given in Section 5 .

\section{2. Experimental Method and Results}

\subsection{PSP measurement technique}

Spatially resolved measurements of adiabatic film cooling effectiveness are obtained by applying a thin coat of UniFIB PSP (Innovative Scientific Solutions Inc.) to the effusion surface. The PSP is comprised of luminescent molecules suspended in a permeable polymer binder. When illuminated by light at the correct wavelength $(380 \sim 520 \mathrm{~nm})$, the luminescent molecules become excited and emit light at longer wavelengths $(620 \sim 750 \mathrm{~nm})$. The principal mechanism affecting light emission is oxygen quenching, whereby the luminescent intensity decreases as the partial pressure of oxygen increases [8]. By using an oxygen-free coolant gas, the measured intensity can therefore be used to infer the local ACE using the heat-mass transfer analogy [6]:

$$
\eta=\frac{T_{\text {main }}-T_{\text {wall }}}{T_{\text {main }}-T_{\text {cool }}}=\frac{C_{O_{2}, \text { main }}-C_{O_{2}, \text { wall }}}{C_{O_{2}, \text { main }}}
$$

For the present work, nitrogen is used as the oxygen-free coolant gas. In this case, the properties of both coolant and mainstream gasses can be considered equal and no correction for molecular mass need 
be applied. However, since the partial pressure of oxygen is sensitive to both pressure and concentration, a correction is required for the local static pressure field across the effusion surface. This is determined from an additional intensity measurement where air is used as both the mainstream and coolant gases.

Using the partial pressures, the ACE can be expressed as:

$$
\eta=1-\frac{P_{\mathrm{O}_{2}, N_{2}} / P_{\mathrm{O}_{2}, \text { ref }}}{P_{\mathrm{O}_{2}, \text { air }} / P_{\mathrm{O}_{2}, \text { ref }}}
$$

where $P_{O_{2}, r e f}$ is the reference intensity measured with no flow.

The luminescent intensity of the PSP is additionally sensitive to temperature and care must therefore be taken to perform the intensity measurements under isothermal conditions $\left(T_{\text {cool }}=T_{\text {main }}\right)[8]$. In practice, this restricts the density ratio between the coolant and mainstream flows to values of $\sim 1$. However, this parameter has previously been shown to have only a small influence on film effectiveness in highly turbulent flows and can be considered second order [20].

\subsection{Wind tunnel facility}

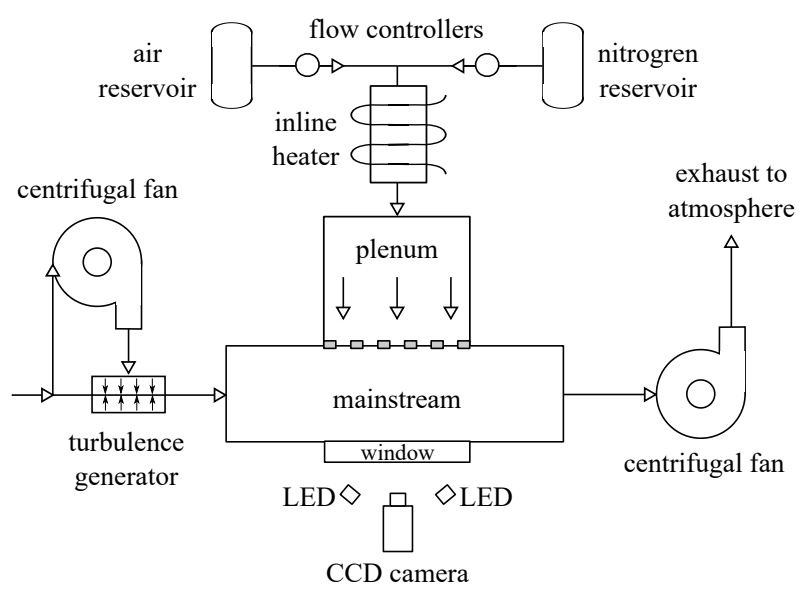

Figure 1: A schematic diagram of the open-loop wind tunnel

ACE measurements are acquired at near-ambient conditions in a continuous flow, open-loop wind tunnel that is shown in schematically in Figure1. The wind tunnel is designed to simulate the flow around a single-skin effusion cooled combustor liner under near-atmospheric conditions. The mainstream flow is provided by a $37 \mathrm{~kW}$ centrifugal fan that draws pre-conditioned air through a working section that is $150 \mathrm{~mm}$ high, $150 \mathrm{~mm}$ wide and $1000 \mathrm{~mm}$ long. By adjusting the fan speed, the velocity within the working section can be increased up to $38 \mathrm{~m} / \mathrm{s}$. Upstream of the working section, an off-take in the tunnel wall diverts some of the mainstream flow to a jets-in-crossflow type turbulence generator. This is provided to vary the bulk intensity level and scale of the fluctuating flow feeding into the test section. The off-take flow is first drawn through a centrifugal fan, before being delivered to a pair of plenums on the tunnel side-walls that reintroduce the flow to the mainstream via perforated plates. The resulting jets create high levels of shear, augmenting the mainstream turbulence levels. Constant temperature anemometry (CTA) has been used to measure the resulting fluctuation characteristics of the flow, giving a rough velocity fluctuation of about $20 \%$. These values are similar to measurements previously made 
in fully-annular combustors at LUTC. With the turbulence generator inactive, the turbulence intensity has been measured at roughly $5 \%$. The lengthscales of the generated turbulent flow are estimated to be from $1 D$ to $20 D$.

Test specimens are designed to be installed in an $90 \times 200 \mathrm{~mm}$ opening in one side of the tunnel working section. The test geometry is a combustor-relevant effusion cooling design that is reproduced at a scale factor of $\sim 4$. The chosen sale factor allows for the inclusion of sufficient effusion holes in the spanwise direction to minimise edge effects, while also permitting the through-hole Reynolds number to be matched to engine conditions. A section through the test specimen used in the current work is shown in Figure 2a The complete specimen is comprised of 9 rows and 9 columns of cylindrical holes in a staggered arrangement, with pitch and space to diameter ratios of 6.2 and an injection angle of $17^{\circ}$. A photograph of the test specimen is shown in Figure 2b.

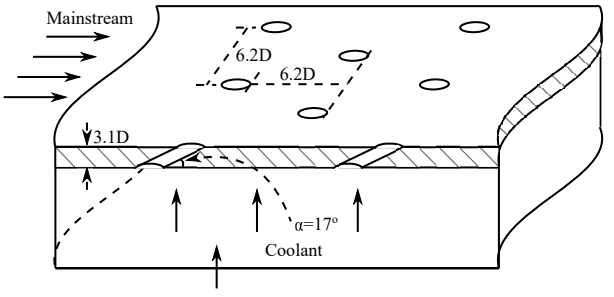

(a) A section with key dimensions

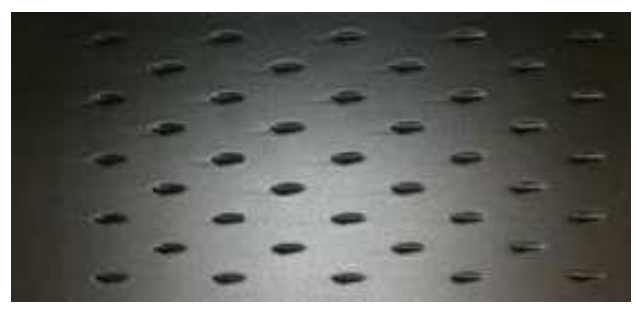

(b) A photograph

Figure 2: The test specimen: (a) A section through the test specimen showing key dimensions, (b) A photograph of the test specimen with cylindrical effusion holes.

Coolant flow is delivered to the test specimen from a plenum that is orientated orthogonal to the working section and is supplied with gas from one of two upstream reservoirs: one air and one nitrogen. Flow from the reservoir first approaches an in-line electric heater that matches the coolant temperature to that of the mainstream. This is necessary to eliminate the influence of temperature variations in the PSP measurements. The flow then passes through an Alicat ${ }^{\mathrm{TM}}$ flow controller, which allows the coolant mass flow rate, and hence pressure drop, to be adjusted. In this way, the through-hole Reynolds number can be matched to engine conditions:

$$
\operatorname{Re}_{D}=\frac{U_{c} D}{\nu}=\frac{\sqrt{\frac{2 \Delta P}{\rho}} D}{\nu}
$$

For the correctly scaled coolant flow, mainstream flow conditions are subsequently set to match the engine momentum flux ratio,

$$
M R=\frac{\rho_{c} U_{c}^{2}}{\rho_{\infty} U_{\infty}^{2}}
$$

The momentum ratio (MR) scales the dynamics of the interaction between the mainstream flow and the coolant jets, which is the most important driver for ACE at high blowing ratios (BRs) 21]. At low BRs, where the flow is attached to the plate surface, the coolant film performance is more dependant on the blowing ratios [22]. Ideally, both momentum and blowing ratios would be matched to engine conditions. However, this relies on maintaining the correct density ratio, which is not feasible under the 
isothermal conditions necessary for the PSP measurements. Table 1 summarises the test conditions in the present experimental investigation. The MRs (BRs) are chosen to achieve test conditions equivalent to engine liner pressure drops $(\Delta P / P)$ of $0.5 \%$ to $2.5 \%$. To examine the effects of free-stream turbulence on $\mathrm{ACE}$, tests at each MR (or BR)are performed with both the turbulence generator on $(T u=20 \%)$ and off $(T u=5 \%)$. The hole-diameter-based mainstream and coolant Reynolds number are at the level of $10^{3} \sim 10^{4}$.

Table 1: A summary of the test conditions

\begin{tabular}{lc}
\hline \hline Parameters & Ranges \\
\hline$U_{c}(m / s)$ & $55.2 \sim 120$ \\
$U_{\infty}(m / s)$ & 32 \\
$\mathrm{MR}$ & $3.11 \sim 15.85$ \\
$\mathrm{BR}$ & $1.81 \sim 4.08$ \\
$\mathrm{DR}$ & 1.05 \\
\hline \hline
\end{tabular}

\subsection{Data acquisition and reduction}

Optical access to the tunnel working section is provided by a large float glass window that is mounted opposite the test specimen. Images of the effusion surface are recorded using a PointGrey Grasshopper 2 GigE machine vision camera with a Sony ICX625 CCD sensor. This allows a maximum image resolution of $2448 \times 2048$ pixels at up to $15 \mathrm{fps}$ with a 14 -bit ADC. A bandpass filter with a $660 \mathrm{~nm}$ centre wavelength and a $30 \mathrm{~nm}$ passband is fitted to the camera lens to eliminate contributions to the measured signal from wavelengths outside the PSP emission range. Illumination is provided by two LED arrays (Luxeonstar Tri-Star) with an emission wavelength of $447.5 \mathrm{~nm}$, which is close to the peak emission wavelength of the PSP. The LEDs are affixed to the camera, ensuring that the illumination field cannot move relative to the camera field of view.

For each measurement condition, 200 images are collected and averaged to calculate the time average intensity fields. A dark image is subtracted from each illuminated image in order to account for the background noise of the camera. Based on the time average intensity fields, the reference image (zero flow) is divided by the two wind-on images (air, $N_{2}$ ) to give pair of intensity ratios $I_{r e f} / I$ for each pixel. Using the modified Stern-Volmer relationship (Eq 5 ), these ratios are related to partial pressures which are then used to calculate ACE from Eq. 2.

$$
\frac{P_{\mathrm{O}_{2}}}{P_{\mathrm{O}_{2}, \text { ref }}}=\frac{C_{\mathrm{O}_{2}}}{C_{\mathrm{O}_{2}, \text { ref }}}=A(T)\left(\frac{I_{r e f}}{I}\right)^{2}+B(T)\left(\frac{I_{r e f}}{I}\right)+C(T)
$$

where the coefficients $\mathrm{A}, \mathrm{B}$ and $\mathrm{C}$ are determined by calibration a priori. Details of the calibration procedure can be found in 23$]$.

\subsection{Uncertainty analysis}

Applying the method of Kline and McClintock [24], the uncertainty in ACE is estimated at $\pm 3 \%$ for $\eta \approx 0.75$, rising to $\pm 35 \%$ for $\eta \approx 0.2$ (at $95 \%$ confidence). The main contributor to this uncertainty is 
the signal-to-noise ratio (SNR) of the camera. Due to the inverse relationship between partial pressure and intensity, $\sim 65 \%$ of the sensor's dynamic range is used to capture low oxygen concentration regions that relate to effectiveness values $>0.8$. As a result, any noise in the signal leads to large uncertainties in regions of high oxygen concentration and low effectiveness. Other uncertainty sources attributable to PSP are detailed by Liu and Sullivan [25], including illumination stability, photodegradation and spectral leakage. However, due to the process of taking the ratio of images and their low contribution to overall uncertainty, many these sources can be neglected.

\subsection{Adiabatic coolant-film effectiveness at different conditions}

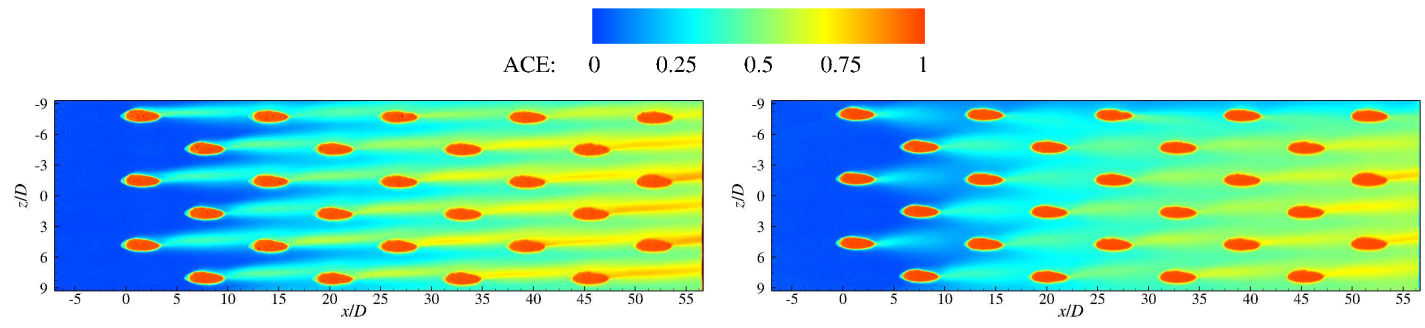

(a) $B R=1.81, T u=5 \%$

(b) $B R=1.81, T u=20 \%$

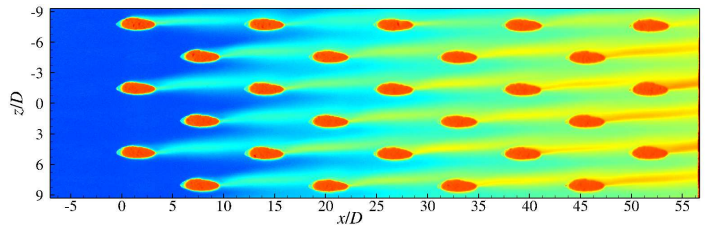

(c) $B R=2.56, T u=5 \%$

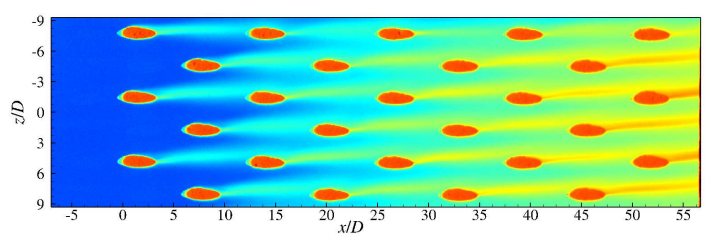

(e) $B R=3.14, T u=5 \%$

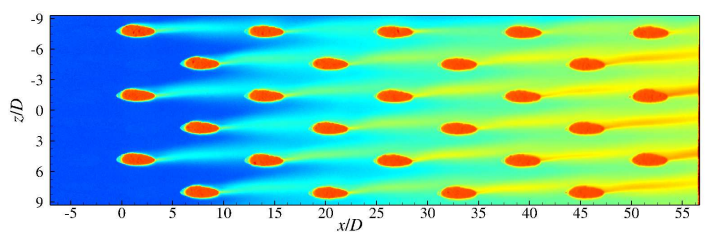

(g) $B R=3.64, T u=5 \%$

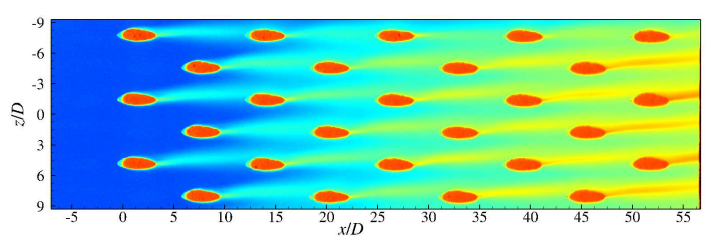

(i) $B R=4.08, T u=5 \%$

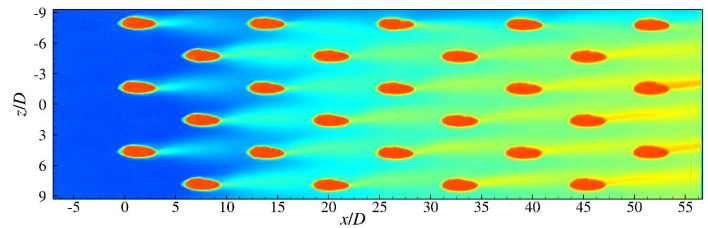

(d) $B R=2.56, T u=20 \%$

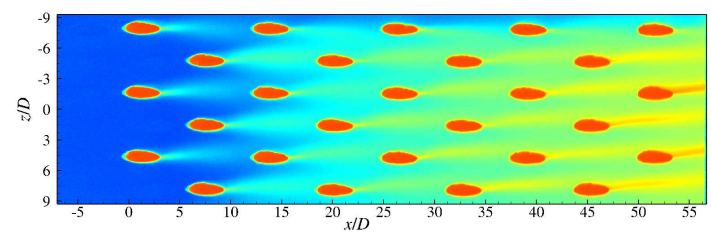

(f) $B R=3.14, T u=20 \%$

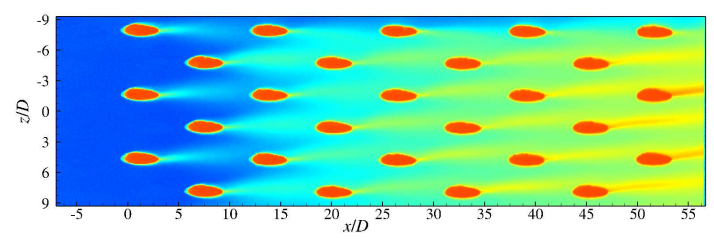

(h) $B R=3.64, T u=20 \%$

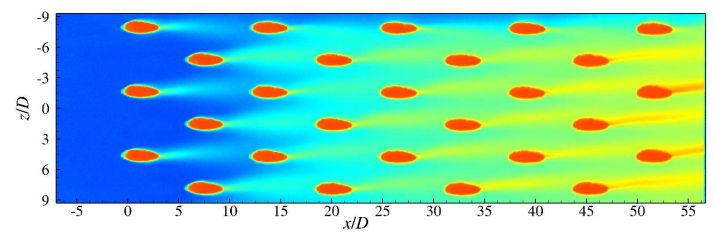

(j) $B R=4.08, T u=20 \%$

Figure 3: Contours of time-mean surface ACE at different BR and $T u$ from experiments

Figure 3 presents contours of surface ACE at different BR and Tu values from the experiment. Due to the additive effect of the jets, the coolant film spreads in the spanwise direction and thickens in the 


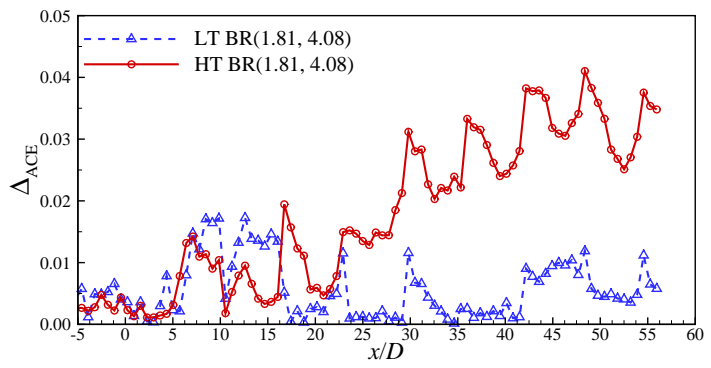

(a) Between $B R=1.81$ and $B R=4.08$

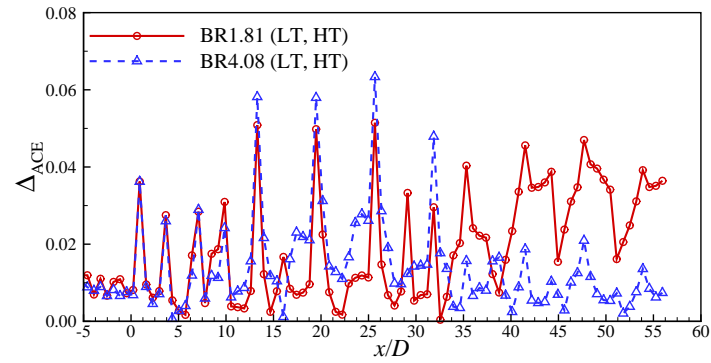

(b) Between LT and HT

Figure 4: Profiles of spanwise averaged time-mean surface ACE difference between measured cases

streamwise direction with increasing distance downstream. The spanwise averaged ACE asymptotes towards values of between 0.55 and 0.6 , with the effectiveness rising for the higher BR conditions. These

\section{Numerical Setup}

\subsection{The flow solver}

The current study employs an in-house hybrid LES solver for unstructured grid, FLUXp (Flux Limited Unstructured eXtrapolation in parallel) [27-32]. In this solver, the Favre-averaged compressible Navier-

Stokes equations for ideal gas are solved in conservation form. A preconditioned formula with a pseudo

values are in reasonable agreement with data published by Schultz at BR of 2 and 3 for a staggered array of cooling holes with a $17 \mathrm{deg}$ injection angle. Although Schlutz's [26] data overlaps the range of BR considered in the present study, this does not account for differences in density and hence momentum ratios between the two sets of results. Considering the influence of inflow turbulence, it can be seen that higher mainstream turbulence intensity leads to a faster break down of the coolant jet and wider spreading in the spanwise direction at a fixed BR. This effect is consistent from low to high BRs, although the effect is greatest at lowest BR. This trend is also noted by Martin [4, 20]. More detailed comparison on the effects of mainstream fluctuation and BR on ACE distribution is carried out by examining the difference between the spanwise averaged ACE profiles as shown in Figure 4 The spanwise averaged ACE of selected 2 cases is compared and the difference in ACE at the same location is calculated. Figure 4 only $1 \%$ difference in $\mathrm{ACE}$ between $B R=1.81$ and $B R=4.08$. However, the effects of BR increased for the high turbulence intensity cases, in which a $4 \%$ is obtained after the 9 th hole. This suggests that increasing the amount of coolant (or momentum of the coolant) will improve ACE in high upstream fluctuation scenarios, but would not be effective at low level of upstream fluctuation. Figure 4 (b) reveals the effects of turbulence intensity at the lowest and highest BRs. At the lowest BR, there's a $1 \sim 2 \%$ difference in ACE values up to the 6 th row $(x / D \approx 35)$, beyond which a much higher difference $(4 \%)$ is presented. At the highest BR, the difference in ACE between the low and high turbulence cases remain a constant level $(1 \sim 2 \%)$ throughout the plate. Again, similar trends were identified by Martin[4, 20]. This suggests that the influence of upstream fluctuation level on ACE is greater at low BR compared with high BR. 
time term is used to overcome the low Mach numerical stiffness, help maintaining the time accuracy and converting the conservative variables $\mathbf{W}=\left[\rho, \rho u_{i}, E\right]^{T}$ to primitive variables $\mathbf{Q}=\left[p, u_{i}, T\right]^{T}$. The inviscid and viscous fluxes are defined as $\mathbf{F}_{i}=u_{i} \mathbf{W}+\left[0, \delta_{1 i} p, \delta_{2 i} p, \delta_{3 i} p, u_{i} p\right]^{T}$ and $\mathbf{G}_{i}=\left[0, \tau_{1 i}, \tau_{2 i}, \tau_{3 i}, \tau_{k i} u_{k}+q_{i}\right]^{T}$. More details of the solver, such as the discretisation and LES-RANS hybridisation can be found in [31, 32].

Two Reynolds-averaged Navier-Stokes (RANS) simulations are also carried out in comparison with the hybrid LES simulations. The RANS simulations are using Menter's SST models [33, 34]. This is the one of the most well-developed and commonly used RANS models, and is specifically developed for wall-bounded flow and flow with adverse pressure gradient.

\subsection{Configuration}

A sketch of the computational domain is illustrated in Figure 5a, It contains only two columns of the coolant holes with a spanwise distance of $3.1 \mathrm{D}$. Using the periodic boundary conditions at the two sides of the mainstream channel, this section can be extended periodically in the spanwise direction, mimicking the full specimen used in the experiments. The top surface of the main channel is located at about 7.75 $\mathrm{D}$ away from the plate surface and it is defined as far-field to allow flow penetration through the boundary. The origin of the computational domain is set at the centre of the two rows and parallel to the leading edge of the first coolant hole. The inlet is about 11.6 D away from the leading edge of the first hole and the outlet is about $15 \mathrm{D}$ away from the trailing edge of the last hole. The bottom surface of the main channel, together with the coolant hole surface and top surface of the coolant chamber, is set as no-slip and zero heat flux wall. This ensures that no extra heat is introduced to the domain and that the wall surface is adiabatic. The coolant holes stretch $3.1 \mathrm{D}$ in the vertical direction with an inclination angle of $17^{\circ}$ to the plate surface, which leads to a length-to-diameter ratio of about $10.6 \mathrm{D}$. The coolant enters the chamber from the bottom surface that is about $4.65 \mathrm{D}$ away from the coolant hole inlet. The surfaces of the 4 sides are slip walls to make sure that all the coolant that enters the chamber will exit from the coolant holes.

The mainstream bulk velocity is $U_{\infty}=32 \mathrm{~m} / \mathrm{s}$ with a velocity profile specified at the inlet. The coolant speed at the inlet of the chamber is adjusted so that a bulk velocity of about $U_{c}=55.2 \mathrm{~m} / \mathrm{s}$ is reached at the coolant hole exit. The mainstream temperature is $T_{\infty}=315 \mathrm{~K}$ while the coolant flow has a temperature of about $T_{c}=300 \mathrm{~K}$. This leads to a density ratio of 1.05 , which is equivalent to the experiments. The blowing ratio of the current case is $\mathrm{BR}=1.81$ and the momentum ratio is about $\mathrm{MR}=3.11$. The reason of choosing this blowing ratio is because it results in a more obvious difference in the measurement between the low and high turbulence intensities. But the understanding of the differences in flow behaviour of both cases is limited from the measurements.

A velocity profile is used at the inlet of the mainstream with synthetically generated free-stream turbulence by SEM 35 38]. Two fluctuating upstream conditions are investigated: a low fluctuation level of $T u \approx 5 \%$ and a fluctuation level of $T u \approx 20 \%$. The two cases are referred to as LT and HT. The integral lengthscale of the velocity perturbation is set to $2 D$, which is within the range of the measured lengthscale range in the experiment but most suitable for the computational domain. The integral lengthscale is kept constant between the LT and HT cases so that the differences between the 

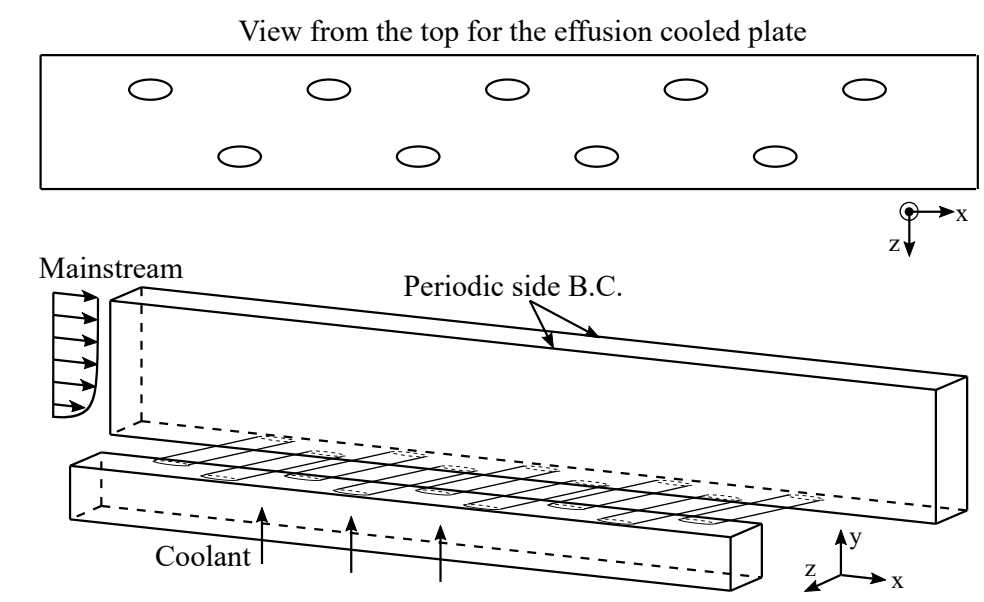

(a) A sketch of the computational domain and boundaries

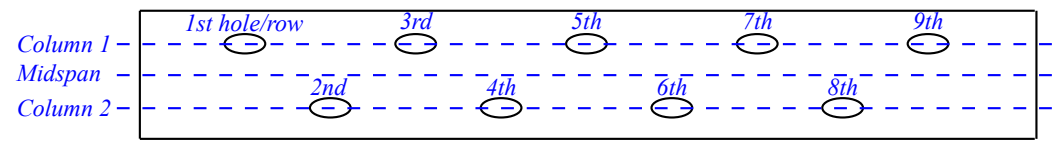

(b) Definition of the coolant hole locations

Figure 5: A sketch of the computational domain and the effusion-cooled plate

simulations are purely due to the turbulence intensity.

An unstructured grid of 18-million cells is generated for the multi-row effusion cooling simulations as Figure 6 shows. The left and right boundaries in the spanwise direction of the mainstream channel are set as cell-to-cell match. This is to comply with the requirement of the periodic boundary condition. Two large refinement zones are applied to cover regions from the upstream of the first coolant hole to the downstream of the last hole of the two columns respectively. This refinement aims to better resolve the shear layer and mixing process of the coolant jets and the mainstream. Surface cells are refined close to and downstream of the coolant holes to better resolve the separation and reattachment of the coolant jets. This is because that the small but highly turbulent near-wall structures are vital to the coolant film distribution and surface ACE. Prism layers are generated from the surface with the first cell height set to satisfy $y^{+} \approx 1.0$ based on the mainstream inlet conditions. The mesh is refined in the coolant jet region as well as key regions close to the coolant exits and near-wall. The refinement is carried out based on a separate validation of a single-row configuration at a much higher diameter-based Reynolds number.

A summary of the simulations carried out in the current study is presented in Table 2, in which $N_{\text {cell }} /\left(\operatorname{Re}^{*} N_{\text {hole }}\right)$ is the Reynolds number based cell count per hole, $\Delta_{\text {min }}{ }^{*}$ Re is the Reynolds number based refinement, $T u$ and $L_{T}$ stands for the intensity and lengthscale of the velocity perturbation, $\Delta t$ the time step size and the convective time is defined as $t^{*}=D / U_{\infty}$.

Table 2: A summary of simulations performed in the current study

\begin{tabular}{cccccccc}
\hline \hline Case & Approach & $\mathrm{BR}$ & $N_{\text {cell }} /\left(\mathrm{Re}^{*} N_{\text {hole }}\right)$ & $\Delta_{\text {min }} * \operatorname{Re}$ & $T u$ & $L_{T}$ & $t^{*} / \Delta t$ \\
\hline LT & hybrid LES & 1.81 & 3970.4 & 0.05 & $5 \%$ & $2 D$ & 200 \\
HT & hybrid LES & 1.81 & 3970.4 & 0.05 & $20 \%$ & $2 D$ & 200 \\
\hline \hline
\end{tabular}



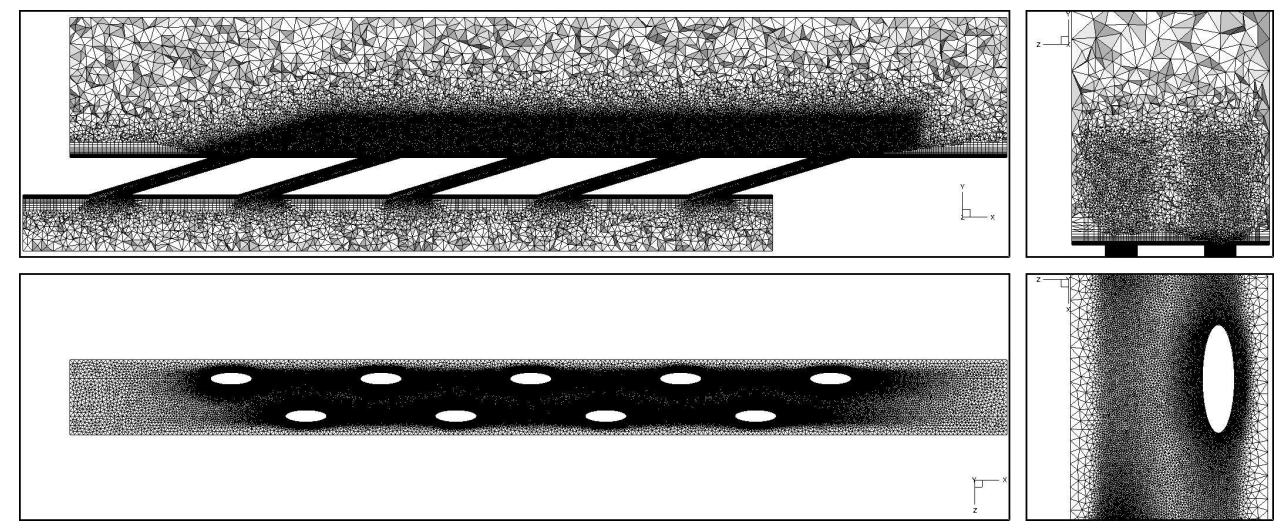

Figure 6: The 18-million-cell grid: a cut-away view through the first column holes (top left), a cut-away view in span (top right), overall view of surface cells (bottom left), enlarged view of surface refinement (bottom right)

\subsection{A validation study}

The numerical methodology is developed and validated on a well-studied and widely-used single-row cooling case carried out by the research group led by D.G.Bogard [21]. Only a brief introduction to the study is shown in this section due to the limited space in this paper and the full text is available in [39]. Simulations are carried out based on three grids with improving grid resolutions, a coarse grid, a medium grid and a fine grid. In the medium and fine grid, the cells are refined in key regions like the coolant exit and near-wall. A brief summary of the single-row simulations are presented in Table 3 . where $N_{\text {cell }} /\left(\operatorname{Re}^{*} N_{\text {hole }}\right)$ is the Reynolds number based cell count per hole, $\Delta_{\text {min }}{ }^{*}$ Re is the Reynolds number based refinement, and $\gamma$ is the blending factor between central difference and upwinding terms.

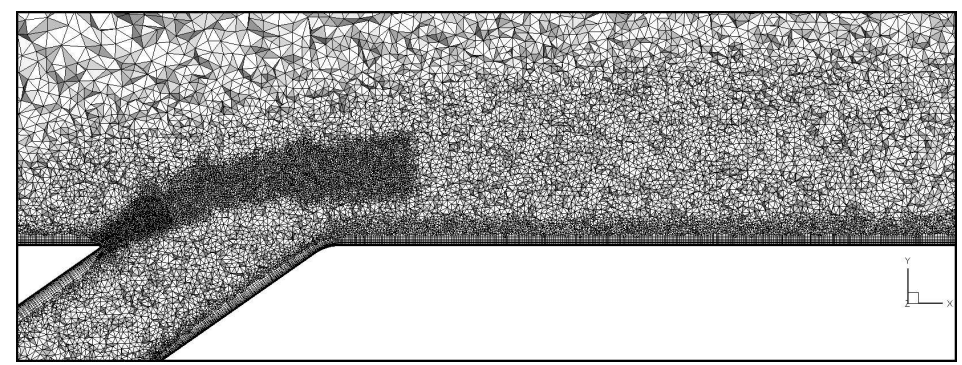

Figure 7: An example of the mesh

Figure 7 presents an example of the mesh with refinement regions. The mainstream bulk velocity is $U_{\infty}=20 \mathrm{~m} / \mathrm{s}$ and the temperature is $300 \mathrm{~K}$. The velocity at the coolant chamber inlet is adjusted so that the bulk velocity at the coolant hole exit is about $U_{c}=10 \mathrm{~m} / \mathrm{s}$ and the coolant temperature is set to $150 K$. This results in a mainstream Reynolds number based on coolant hole diameter of about 16, 000. ${ }_{265}$ The density ratio is $\mathrm{DR}=2.0$ and the velocity ratio is $\mathrm{VR}=0.5$, thus the blowing ratio is $\mathrm{BR}=1.0$.

Table 3: A brief summary of simulations carried out in the single-row study

\begin{tabular}{ccrccc}
\hline \hline Case & $N_{\text {cell }} /\left(\mathrm{Re}^{*} N_{\text {hole }}\right)$ & $\Delta_{\min }{ }^{*} \mathrm{Re}$ & \multicolumn{1}{c}{$\gamma$} & $\mathrm{BR}$ & $\mathrm{DR}$ \\
\hline Coarse & 387.5 & 16.256 & $0.5 \sim 1.0$ & 1.0 & 2.0 \\
Medium & 498.0 & 4.064 & $0.5 \sim 1.0$ & 1.0 & 2.0 \\
Fine & 629.0 & 3.048 & $0.5 \sim 1.0$ & 1.0 & 2.0 \\
\hline
\end{tabular}


The time-mean ACE profiles at the centreline $(z=0)$ for the cases with different grid resolutions are presented in Figure 8. The Coarse case underestimates the centreline ACE, but the decreasing trend remains similar. The profiles of the refined-grid cases, Medium and Fine, are in good agreement with the measurements. The comparison of results between Medium and Fine indicates that the surface exit.

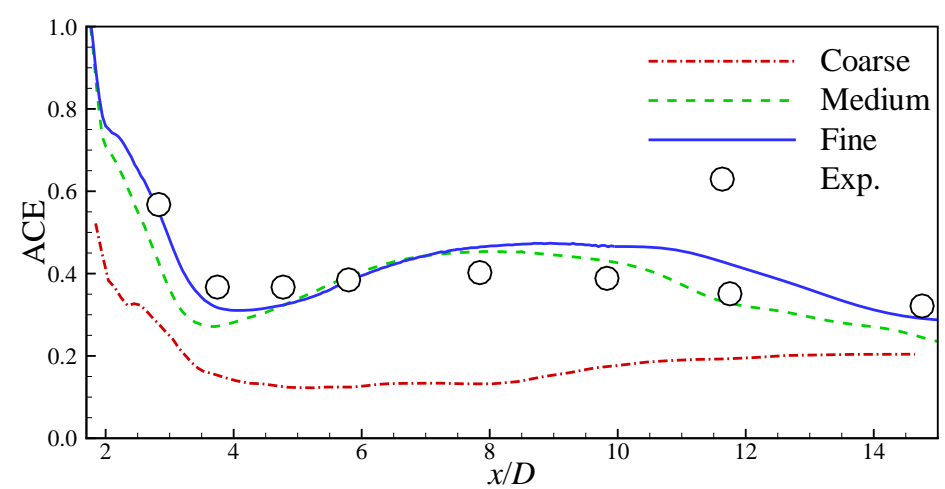

Figure 8: Streamwise distribution of time-mean surface ACE on the centreline $(z=0)$ of the single-row study

\section{Results and Analysis}

\subsection{Examination of adiabatic coolant-film effectiveness}

Figure 9 shows contours of the time-averaged surface ACE of LT and HT cases and the corresponding experimental results. Case LT presents a similar coolant film coverage as the measurements, while the RANS LT underestimates both the coverage and strength of the coolant film. For the first three rows of case LT, the surface ACE after the coolant exit is slightly lower than the measurements. This is due to an underprediction in the amount of coolant that reattaches to the surface. From the 4th row of case LT, an increasing amount of coolant is reattached to the plate surface and the surface ACE increases significantly. However, the strength of the force, which is from the upstream mixed flow and pushes the downstream coolant jets onto the plate surface, is underpredicted. Such underestimation leads to a slightly lower value in the high ACE region near the centreline downstream of each coolant hole at $x>25 D$ of case LT compared to that in the experiment.

In terms of the HT cases, the surface ACE contours of case HT compare favourably with that of the experiment, while RANS HT provides an underestimated the surface ACE. It is worth noting that RANS HT gives a better prediction on the formation of the coolant film compared to RANS LT, which can be contributed to the high inflow turbulence activating the RANS turbulence model. Comparing LT and HT contours, it can be found that the coolant reattaches to the plate surface earlier in the wake of 


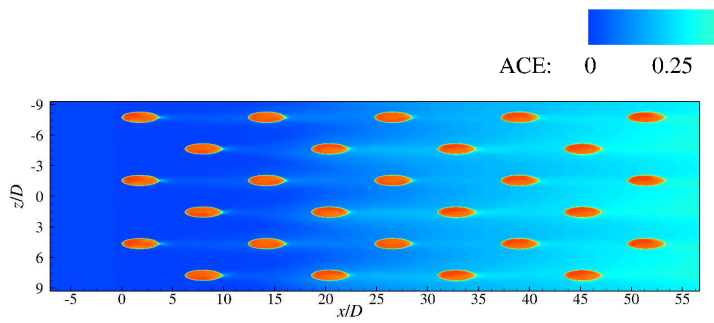

(a) RANS LT

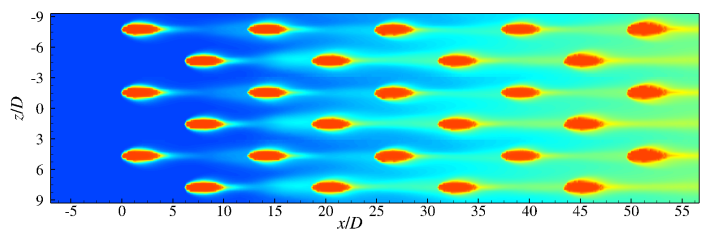

(c) case LT

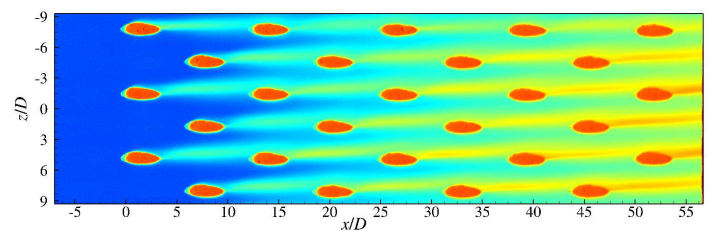

(e) Experiment LT

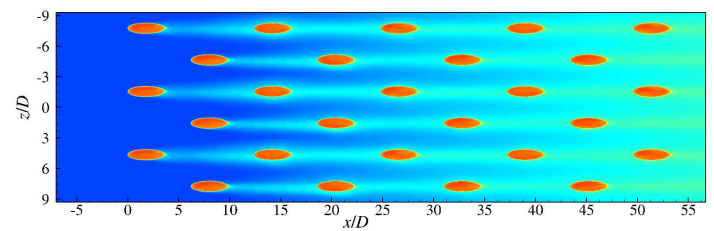

(b) RANS HT

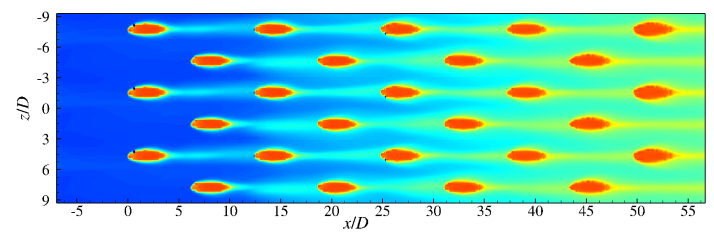

(d) case HT

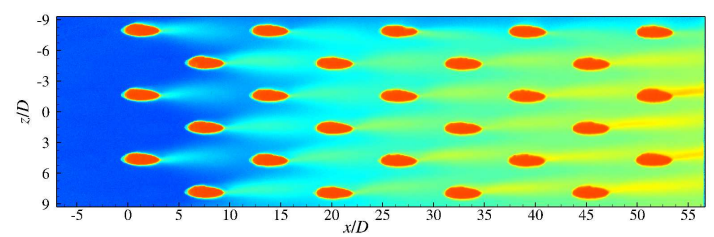

(f) Experiment $\mathrm{HT}$

Figure 9: Contours of time-averaged surface ACE at $M R=1.81$, LT cases on the left column, HT cases on the right column

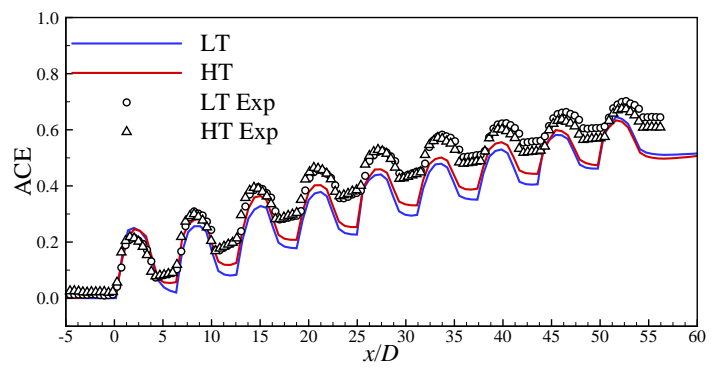

Figure 10: Spanwise-averaged time-averaged surface Fig $\mathrm{ACE}$

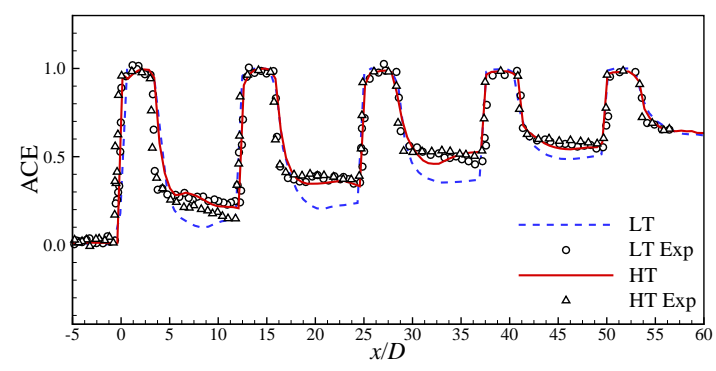

Figure 11: Streamwise distribution of time-averaged surface ACE through the centreline of column 1 holes

the first 3 rows at high inflow turbulence intensity condition. This leads to a higher surface ACE and a wider coolant film coverage across the span of the first 3 rows. The reduction in surface ACE at the downstream edge of the coolant jet after the first coolant hole is also captured by the simulation. The reason for this early reduction is that the high turbulence intensity enhances the mixing and spanwise spreading of coolant and thus leads to a rapid increase in coolant film temperature.

The spanwise- and time-averaged ACE profiles of LT and HT cases are shown in Figure 10, The ACE profiles of cases LT and HT agree reasonably with the measurements. The increasing trend in surface ACE after each hole is captured in both cases. It is found that peaks in the ACE profile occur every $6.2 D$. The occurrence of these peaks is due to the inclusion of data inside the coolant hole, which is almost constant at 1.0, during the spanwise averaging process. Hence, it results in much higher spanwiseaveraged values at streamwise positions where the coolant hole is presented. A small underestimation of the surface ACE occurs in the downstream region of $x>10 D$, indicating the amount of coolant 
reattaching to the plate surface being underpredicted. One of the reasons might be in the resolution of the very-small-scale near-wall turbulent structures is not high enough. These structures, which consist of the upstream turbulence, coolant reattachment, coolant-mainstream mixing etc, bring the coolant and hot mainstream together and thus determine the temperature and coverage of the coolant film near the wall. It might also be due to the hole geometry, surface roughness and inflow conditions as the near-wall mixing is extremely sensitive to those conditions. Furthermore, it is found that the spanwiseaveraged data averaged out one of the significant effects of the turbulence intensity that is shown in the ACE contours, which is the wider coolant spreading and the early decay caused by the higher inflow turbulence.

The streamwise distribution of the ACE through the centreline of the first coolant hole column is illustrated in Figure 11. A higher ACE value in the near-hole region downstream the coolant exit is obtained in case HT as Figure 11 shows. This is because of the coolant jet reattaching to the surface earlier due to the higher intensity level in case HT as shown in Figure 16. However, the ACE value decays faster in case HT compared to case LT, as the high turbulence intensity increases the mixing between the coolant film and mainstream. It is noted that the experiments show a close value between the two turbulence level cases. This may due to differences in conditions between the experiments and simulations, for example the fluctuating inflow generated by the wind tunnel may not be exactly reproduced by the synthetically generated one in the simulations, and the laser-drilled coolant holes with a rough surface in the experiment may result in a wider spread and faster reattachment of the coolant. In addition, the enhancement in surface ACE in the high intensity case reduces from the 1st coolant jet to the last. This is possibly because the coolant jets from the downstream holes are squeezed to the surface by the mixed mainstream flow, which consists of the mainstream and upstream coolant jets. Therefore, the effects of forced attachment become more evident than the effects of the mainstream turbulence in determining the coolant film performance and surface ACE distribution after the 6th row.

330 The measurements show that the ACE value after the first peak in case LT is slightly higher than that in case HT. The reason can be found from the ACE contours that the increased mixing of coolant and mainstream due to the high turbulence intensity leads to a much faster decay in ACE downstream of the coolant exit. The effect of turbulence intensity diminishes after the 2nd hole in the experiments, which is earlier than that in the simulation.

\subsection{Examination of the coolant film thickness}

Downstream the coolant holes, a flow region attached to the plate surface, where the local flow temperature is much lower than the mainstream, is formed due to the mixing of the coolant jets. This low temperature region is referred to as the coolant film, which protects the surface from the high temperature mainstream. This region is three dimensional and a clear boundary is present between this region and the mainstream as a result of heat transfer. The distance from the boundary to the plate surface is regarded as the coolant film thickness in this paper. In order to quantify the thickness at different positions, a temperature recover factor is used to calculate the locate the boundary which is 
similar to the velocity recovery factor used in the boundary layer theory [40],

$$
F_{T}(\mathbf{x})=\frac{T(\mathbf{x})-T_{c}}{T_{\infty}-T_{c}}
$$

in which $F_{T}(\mathbf{x})$ denotes the temperature recovery factor at a specific point in the flow domain. If the temperature recovery factor is $1\left(F_{T} \approx 1\right)$ at a selected position, it means that the position is covered by the mainstream. If $F_{T}<1$, the position is being covered by the coolant film. Hence, the boundary between the coolant film and the mainstream can be defined at a given temperature recovery factor.

Figures 12 and 13 illustrate the coolant film by the isosurfaces of temperature difference at $F_{T}=75 \%$ and $F_{T}=99 \%$. It can be found that the first coolant jet is broken down earlier in case HT compared to case LT, and thus breaks up the coolant film of $F_{T}=75 \%$ close to the downstream coolant hole as Figure 13 shows. The isosurfaces at $F_{T}=99 \%$ in both cases show the development of coolant film from the 1st coolant hole, and it suggests that a film that covers the whole plate surface, though the temperature difference is small, is formed downstream the 3rd coolant hole.

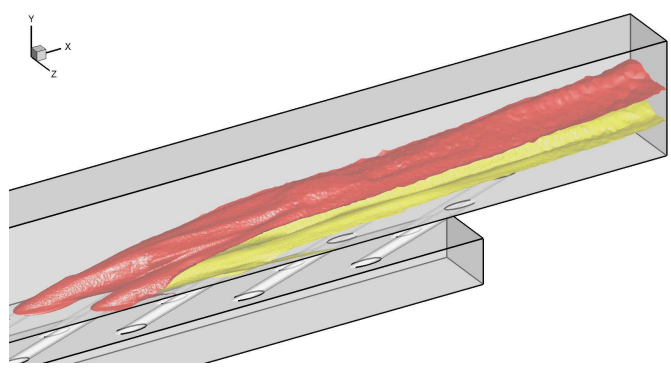

(a) $\mathrm{LT}$

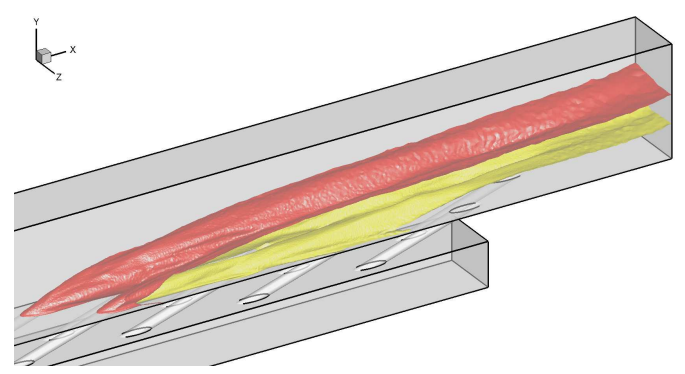

(b) $\mathrm{HT}$

Figure 12: Isosurfaces of the coolant film at $F_{T}=75 \%$ (yellow) and $F_{T}=99 \%$ (red) of case: (a) case LT and (b) case HT

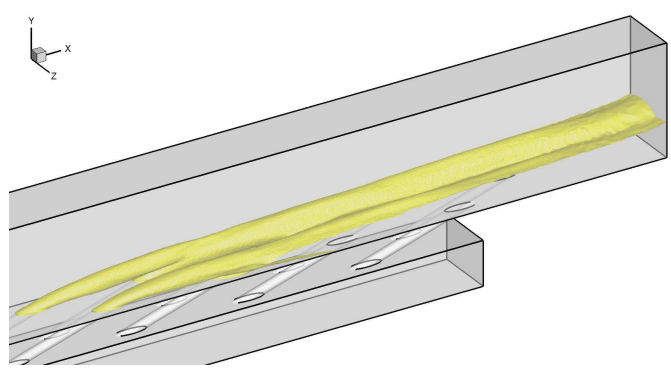

(a) LT

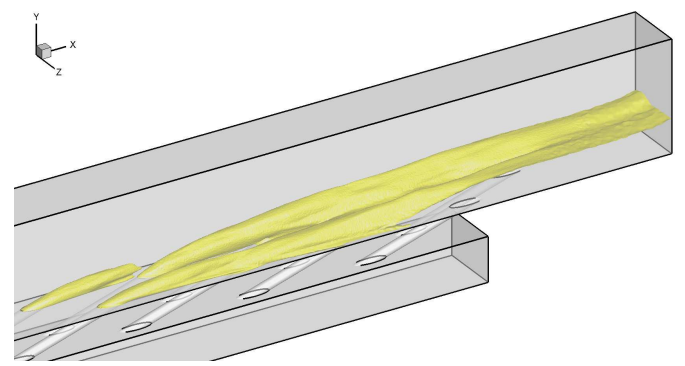

(b) HT

Figure 13: Isosurfaces of the coolant film at $F_{T}=75 \%$ of case: (a) case LT and (b) case HT

To study the position of the coolant film boundary, as well as the coverage in the streamwise and spanwise direction, a coolant film "thickness" can be defined to illustrate the distance between the coolant film boundary and the plate surface. Similar to the velocity boundary layer thickness, which is defined at the position where the velocity recovery factor reaches $99 \%$ [40], the coolant film thickness $\delta_{F_{T}}$ can be defined when the temperature recovery factor reaches $99 \%$,

$$
\delta_{99 \%}=\max \left(F_{T}^{-1}(k=99 \%)\right)
$$


More detailed comparison is achieved by comparing the streamwise and spanwise coolant film thickness distribution as Figures 14 and 15 show. The early break down of the 1st coolant jet in case HT is clearly illustrated in the profiles of $\delta_{75 \%}$ by a drop in the coolant film thickness at about $x=12 D$. The streamwise distribution profiles at both $\delta_{75 \%}$ and $\delta_{99 \%}$ suggest that the high turbulence intensity not only results in an early break down of the coolant jet, but also leads to a slower coolant film thickness growth. It can be concluded that a better spanwise coverage of the coolant film is achieved in case HT compared to case LT in the wake of the first 3 coolant holes. However, the increased flow mixing due to high turbulence intensity brings more hot gas to the plate surface in the middle of the two columns, thus leads to a drop of the coolant film thickness near $x=0$.

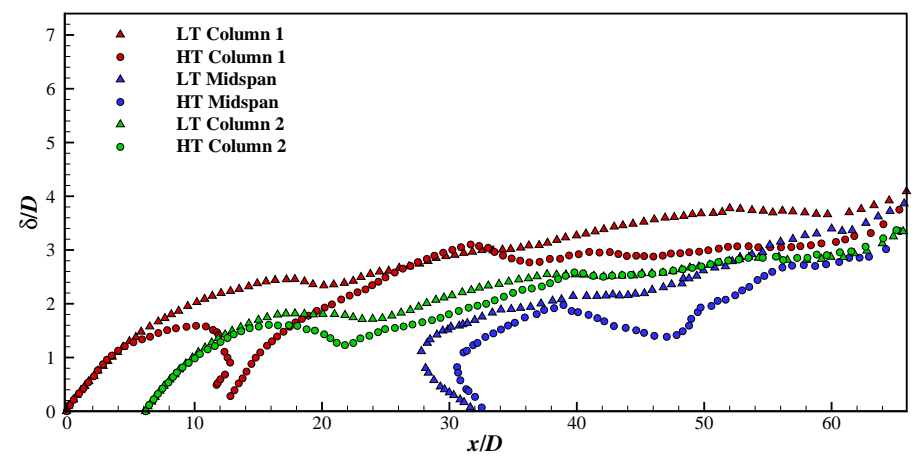

Figure 14: Profiles of coolant film thickness $\delta_{75 \%}$

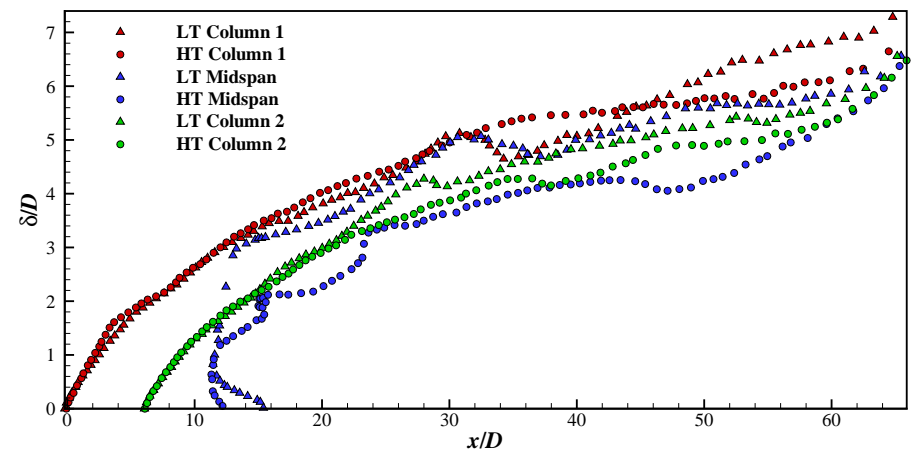

Figure 15: Streamwise coolant film thickness $\delta_{99 \%}$ distribution

\subsection{Examination of the temperature field}

The time-averaged temperature field is studied first to show the behaviour of the coolant jet and the mainstream. Figure 16 illustrates the time-averaged temperature contours at a slice through the first column of holes. Overall, the thickness of the coolant film, denoted by the position of the upper shear layer, is greater in case LT than in case HT. This owes to the jet breakdown and enhanced mixing effects of the inflow turbulence. Due to the high momentum ratio $(M R=3.18)$, the coolant jet from the first hole in case LT does not reattach to the plate surface. This results in a high temperature flow occurring near the plate surface downstream the first hole exit in case LT. Meanwhile, the coolant jet breaks down 
earlier due to the higher turbulence intensity in case HT and the plate surface downstream the first hole exit is better cooled. A decreasing near-wall flow temperature is found clearly in the streamwise direction in case LT. This decreasing near-wall flow temperature can also be found in case HT, although the reduction is less than that in case LT. The coolant flow fully reattaches to the plate surface after the third hole in both cases. It seems that the downstream coolant jets are pressed onto the plate surface by the mixed upstream flow. Furthermore, it can be found that the low temperature jet core of the downstream jets extends further in the streamwise direction. This is possibly due to the increased mass flow and reduced temperature in the mixed flow after a number of coolant jets are injected.

Figure 17 illustrates the coolant jet reattachment to the plate surface. This reattachment increases the mixing of the coolant and hot mainstream on the plate surface and reduces the heat transfer from the hot mainstream to the combustion tile. The accuracy in capturing this reattachment and predicting the mixing effect is of vital importance in predicting the ACE distribution on the plate surface.

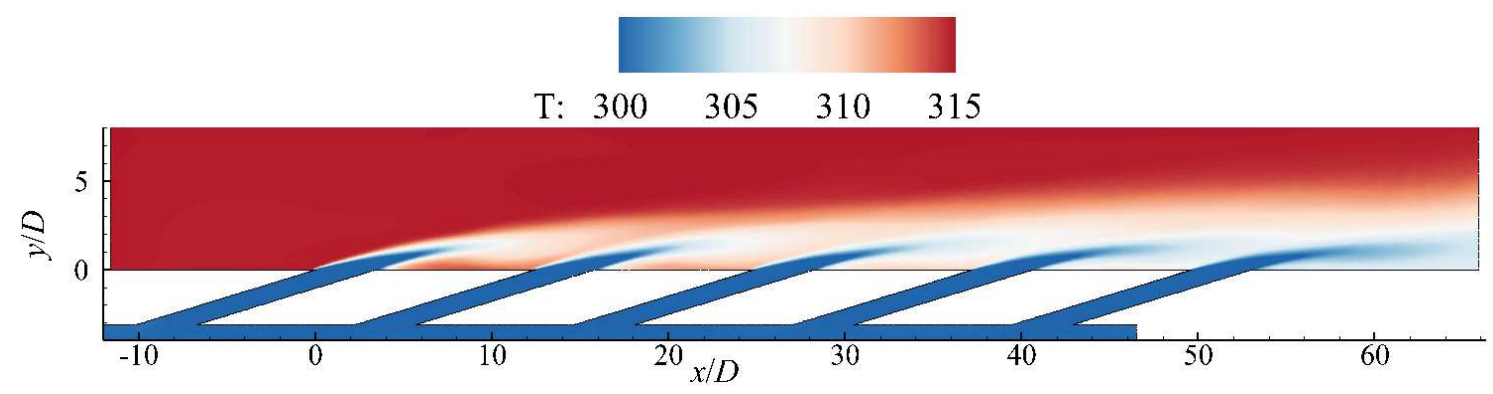

(a) case LT

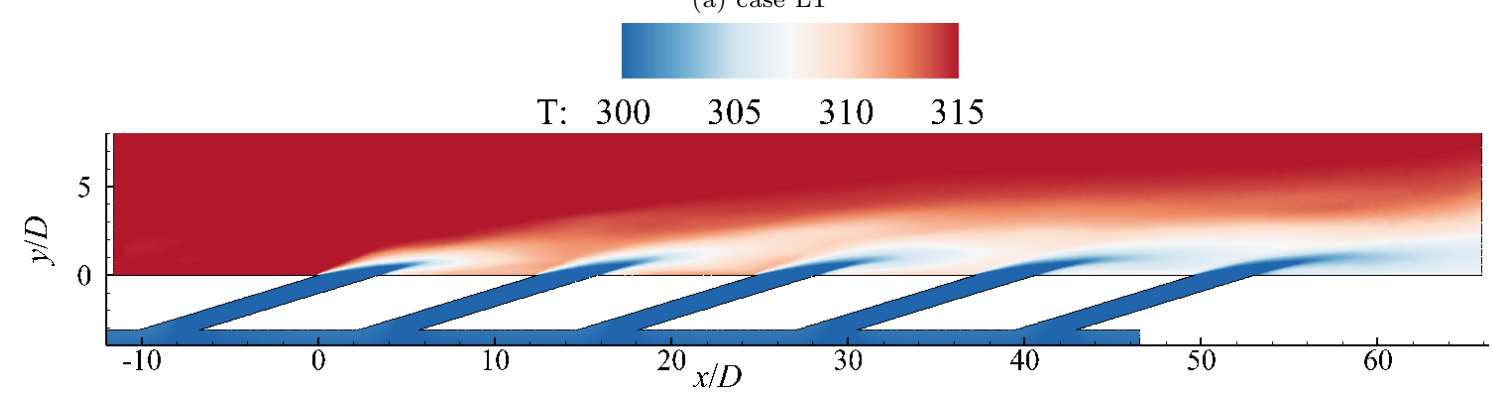

(b) case HT

Figure 16: Time-averaged temperature contours at a slice through the first column holes: (a) case LT, (b) case HT

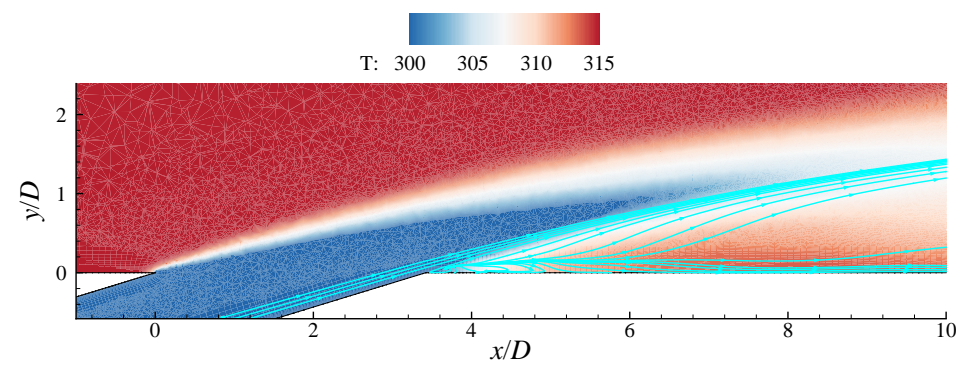

Figure 17: The streamlines of coolant jet reattachment on time-averaged temperature contours at a slice through the first column holes of case LT 


\subsection{Examination of the coolant jet centrelines}

In this section, investigations are carried out to study the differences of the coolant jet injection to the mainstream between the upstream and downstream jets, as well as differences between case LT and case HT. Each coolant jet is represented by the time-averaged path of the coolant flow centreline before it merges with the downstream jet. This coolant flow centreline is determined by the position where the maximum concentration of the coolant flow is presented (or the centre of a maximum concentration region) along the path of the coolant jet,

$$
C_{\max }=\left.\frac{T-T_{\infty}}{T_{c}-T_{\infty}}\right|_{\max }
$$

The resulted time-averaged path of the coolant jets in the 1st column of case LT and case HT are illustrated in Figure 18 .

The coolant jet paths are then aligned to have the same starting point in the streamwise direction as Figure 19 shows. A common trend between the two cases is that the first coolant jet in the column is bent towards the plate surface due to mixing with the mainstream. However, the downstream coolant jet, which is the 3rd coolant jet, penetrates further in the vertical direction. This is because the 1st coolant jet forms a preliminary coolant film that adjust to the coolant velocity and temperature, and this preliminary coolant film reduces the shear stress when the downstream coolant jet injects to the mainstream. However, the limitation on the coolant jet vertical penetration increases when moving further downstream. This limitation is due two reasons. The first reason is the blockage due to more fluid being injected into the mainstream that forces the injected jet to stay attached to the plate surface. The second reason is that a complete coolant film is formed further downstream, with a velocity that is more parallel to the plate surface, and the downstream coolant jet is bent more by the upstream flow once injected to the mixed coolant film.

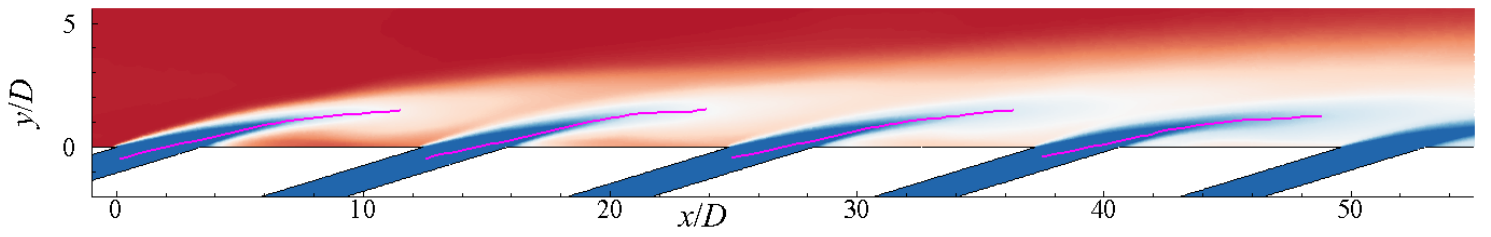

(a) case LT

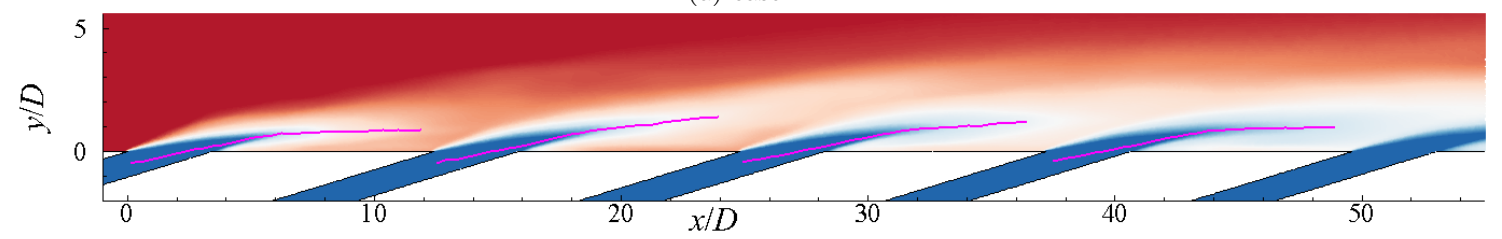

(b) case $\mathrm{HT}$

Figure 18: The 1st column of coolant jet centrelines on contours of temperature: (a) case LT, (b) case HT

Figure 20 presents a comparison of the coolant jet trajectories between case LT and case HT. The high turbulence intensity is increasing the flow mixing and stops the 1st coolant jet penetrating into the mainstream vertically. Also, due to the effect of high turbulence intensity, the 1st coolant jet breaks down not far downstream and thus has little influence on the downstream coolant jet (the 3rd coolant 


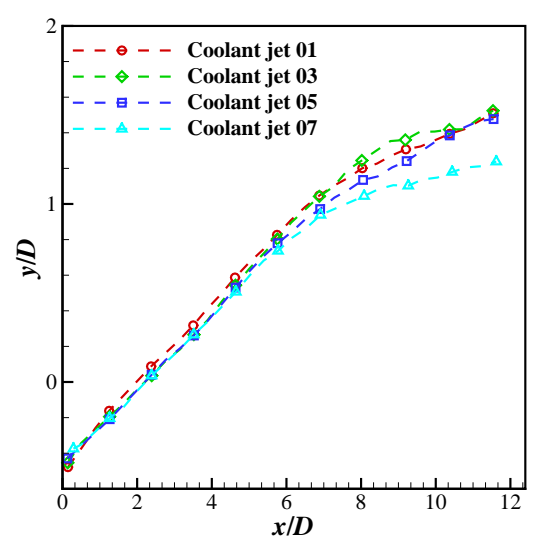

(a) case LT

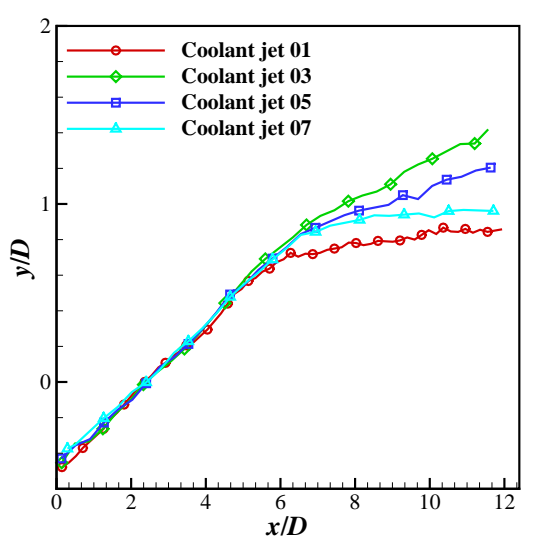

(b) case $\mathrm{HT}$

Figure 19: Aligned coolant jet paths from the 1st column of: (a) case LT, (b) case HT

jet). This explains the reason that the 3rd coolant jet for both cases are the most similar pair among the 4 coolant jets. It can be observed from the 5 th and 7 th coolant jets that the bending of the coolant jets towards the plate surface becomes increasingly effective when more coolant is injected to the mainstream, and the effect of free-stream turbulence becomes less dominant. This is consistent with the behaviour of the ACE distribution and the temperature contours.

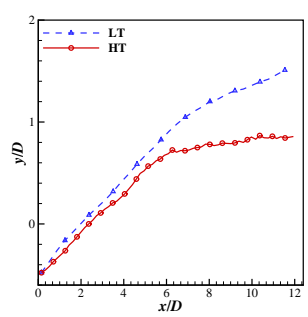

(a) 1st coolant jet

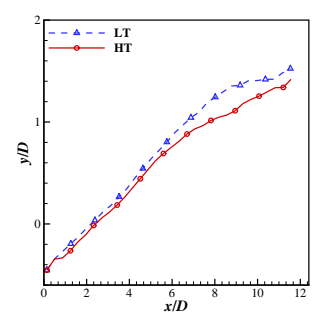

(b) 3rd coolant jet

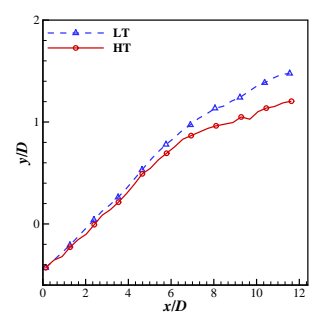

(c) 5 th coolant jet

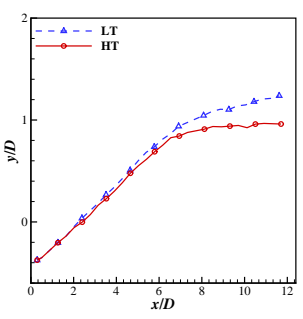

(d) 7 th coolant jet

Figure 20: Comparison of coolant jet trajectories of case LT and case HT

\subsection{Analysis of turbulent structures}

The instantaneous flow features are investigated to study the relation between the turbulent structures and the coolant film distribution on the plate surface. The instantaneous 3D flow structures for case LT are illustrated by isosurfaces of $\lambda_{2}$, and coloured by ACE in Figure 21. The spanwise vorticity contours on a slice through the first column of holes are also presented. It is presented in the figures that the shear layers are formed at the interface between the coolant jet from the first hole in each column and the mainstream. The Kelvin-Helmholtz structures can be observed from the leading edge of the first hole in each column. These structures develop and break down into smaller structures in the downstream region. The hairpin vortices are generated due to the interaction with the plate surface. However, the shear layer of the first coolant jet in each column breaks down much earlier in the multi-row case with turbulent inflow and more small turbulent structures are formed downstream when the mixed coolant flow comes across the jet from the next coolant hole. The mixed mainstream flow will push the downstream coolant 
jets towards the plate surface. This phenomenon has proven to reduce the coolant film temperature attached to the surface and therefore increases the surface ACE. It is also known that the effect becomes more dominant when it moves further downstream, since more coolant jets join the mixed flow. Also, a wider spanwise spreading of the coolant film is obtained as the downstream coolant jet is squeezed to the plate surface.

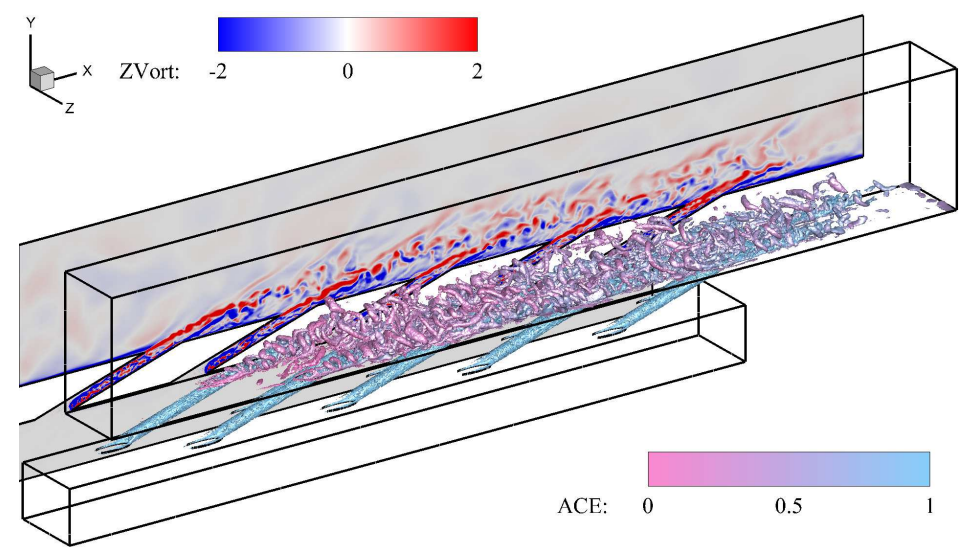

(a) case LT

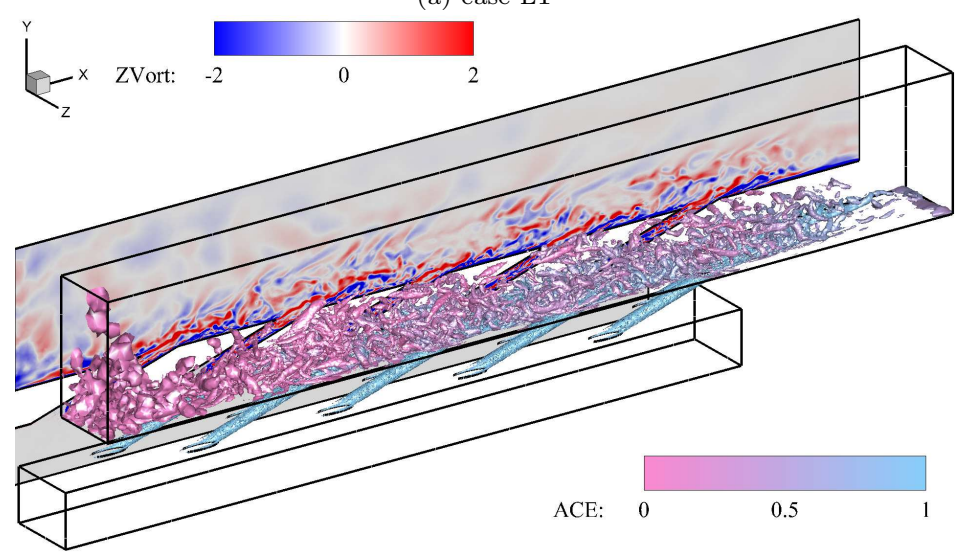

(b) case HT

Figure 21: 3D flow structures illustrated by $\lambda_{2}$ and coloured by temperature

The instantaneous temperature contours on a slice through the first column of holes are presented in Figure 22. The turbulent inflow, jet-mainstream shear layers and smaller structures caused by the separation and reattachment of the coolant flow are clearly illustrated in the contours of vorticity. The effects of these structures on the near-wall flow temperature can be studied from the contours of temperature. As Figure 22 shows, the shear layer formed at the leading edge of the first hole is broken down by the inflow turbulence not far downstream the trailing edge of the coolant hole. Thus, the coolant jet penetration into the mainstream is shortened. This leads to a faster reattachment of the coolant jet to the plate surface, which would reduce the near-wall flow temperature. However, the inflow turbulence also enhances the mixing of the coolant jet and hot mainstream. As a result of the combination of these two effects, the surface ACE downstream the first 2 holes in Figure 22 remains at a low level. Moreover, the early breakdown of the coolant jet also results in a reduced force in pushing the coolant jet from the downstream coolant hole to the plate surface in this multi-row configuration. Therefore, the surface 
ACE after the 2nd and 3rd coolant holes is still dominated by the breakdown effect of the inflow turbulence. As pointed out by the surface ACE distribution, the squeezing effect of the mixed mainstream flow becomes dominant only after the 6 th row.

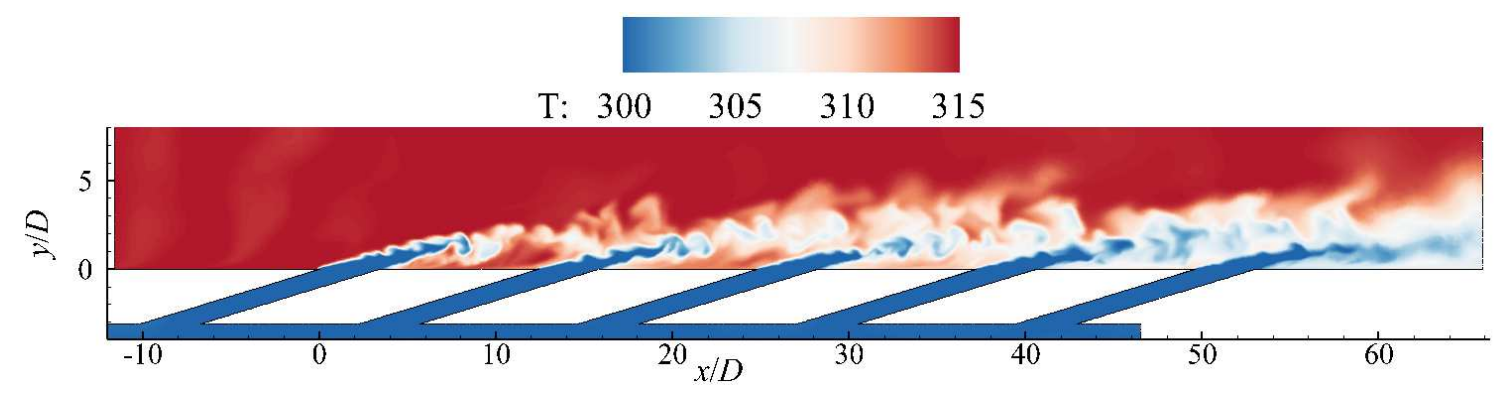

(a) case LT

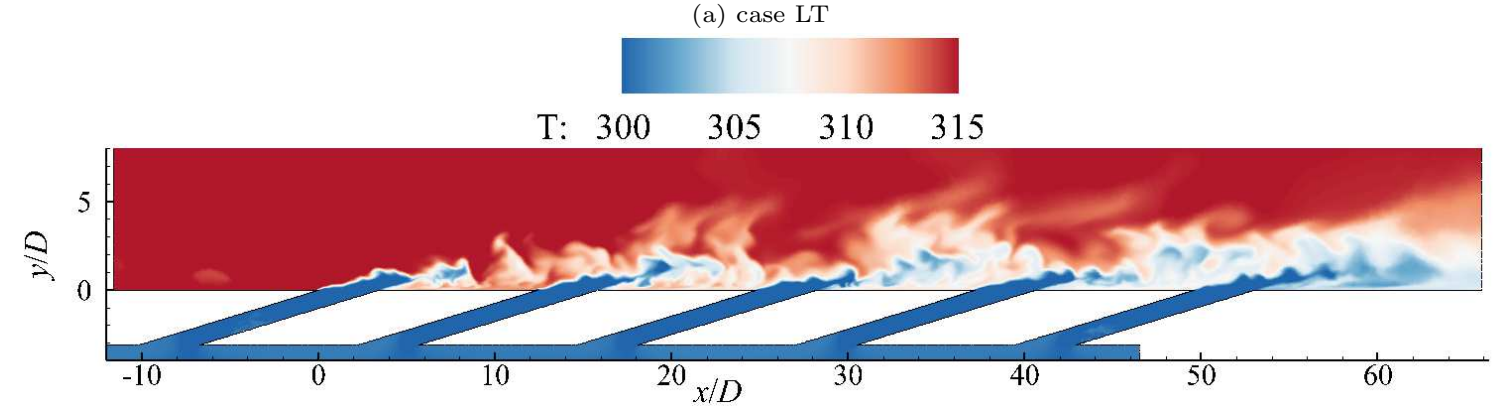

(b) case HT

Figure 22: Instantaneous contours of temperature through the first column holes

\subsubsection{Spectral analysis}

More detailed analysis on the turbulent flow are carried out by examining the power spectra in the upper shear layer and on the wall downstream of the first column of coolant holes. Figure 23 shows a sketch of the probe positions to extract the time signal for the spectral analysis. Two groups of probes are considered. The first group is located in the upper shear layer $y=1 D$ at the trailing edge of each coolant exit. The second group is placed on the plate surface and at the same streamwise position as the first group.

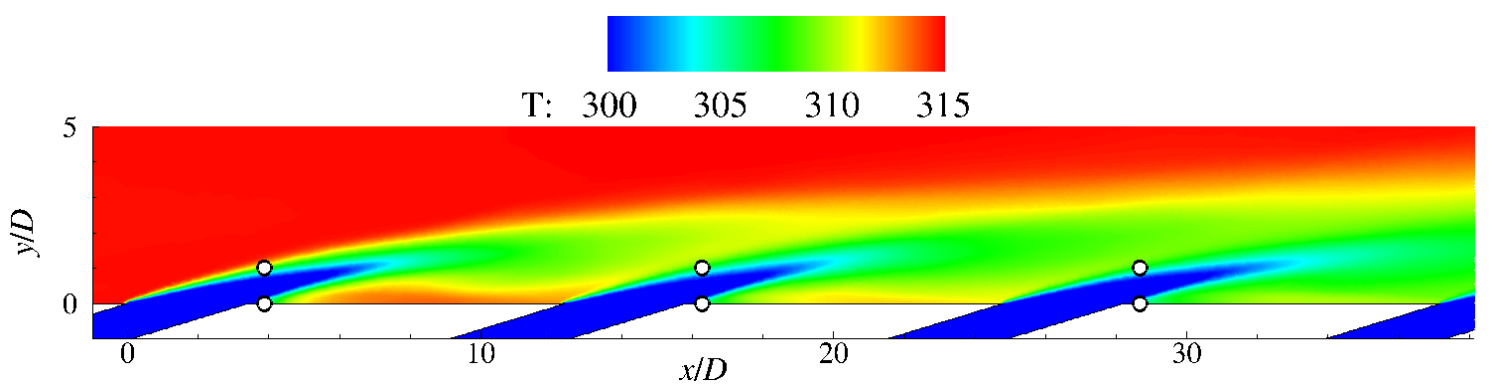

Figure 23: A sketch showing the location of the probes for the spectral analysis

The spectra of temperature in the upper shear layer $(y=1 D)$ and on the wall of case LT are presented in Figure 24, while those of case HT can be found in Figure 25. In the spectra of case LT, it is clearly shown that the temperature spectra both in the shear layer and on the wall have a major peak frequency at about $8000 \mathrm{~Hz}$, which is believed to be the shear layer instability frequency. The second and third 


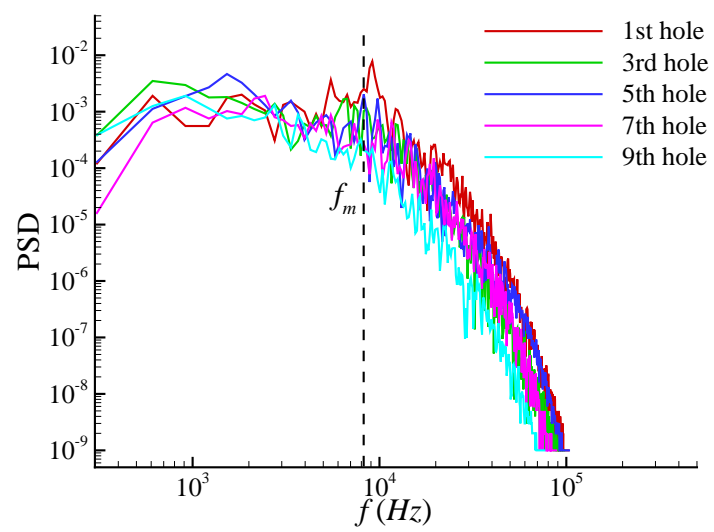

(a) Shear layer

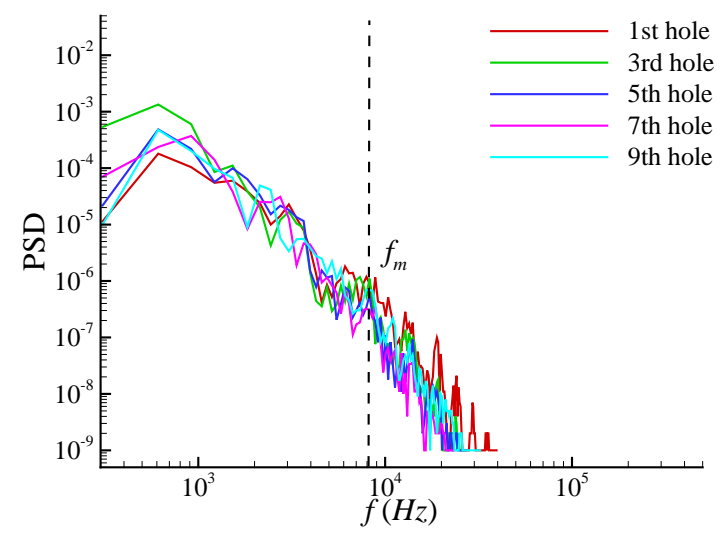

(b) Wall

Figure 24: Power spectral density of temperature (a) in the shear layer and (b) on the wall of case LT

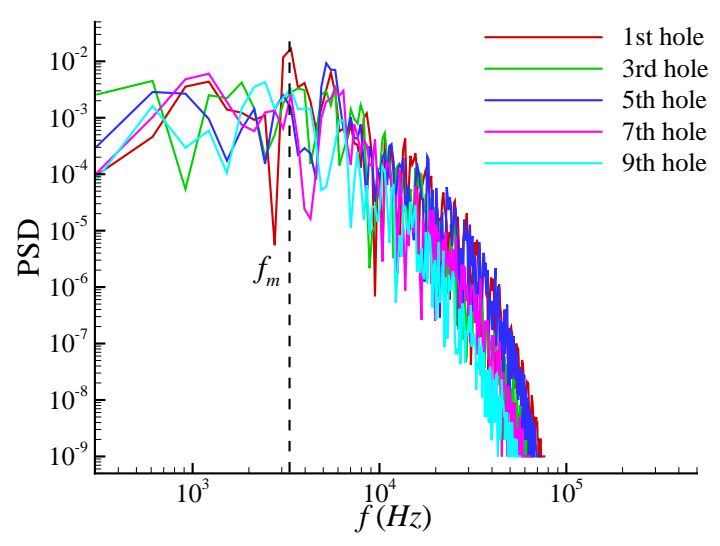

(a) Shear layer

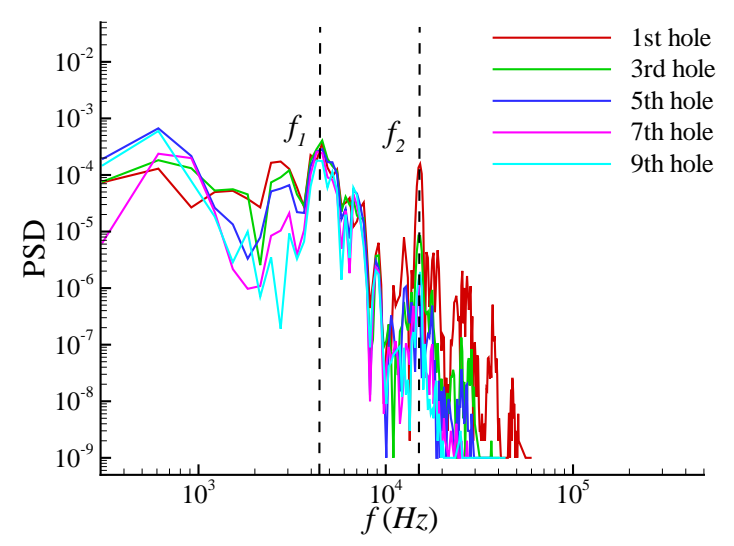

(b) Wall

Figure 25: Power spectral density of temperature (a) in the shear layer and (b) on the wall of case HT

peaks are also found in the spectra of the first hole both in the upper shear layer and on the wall. This is because the upstream flow for the first coolant jet does not contain any mixed coolant flow. Therefore, the instability in the shear layer is purely generated by the mixing of coolant jet and mainstream. Moreover, the presence of lower shear layer also brings a few frequency modes to the temperature spectrum on the This is because the coolant injected to the mainstream from the upstream holes results in a reducing temperature of the mixed flow. Therefore, the temperature fluctuation becomes smaller in the position where the upper shear layer should be. In case HT, the temperature spectra in the shear layer also have a major frequency close to the major shear layer unsteady movement frequency $4000 \mathrm{~Hz}$. However, the surface temperature is more turbulent compared to case LT. This is due to the earlier breakup of the coolant jet upper and lower shear layer enhancing the mixing between the hot mainstream and cold coolant jet close to the plate surface. All these effects are contributed to the higher turbulence intensity in the inflow. Therefore, it is clearly shown that there are two very strong modes in the surface temperature fluctuation spectra: one at around $f_{1} \approx 4000 \mathrm{~Hz}$ corresponding to the major upper shear layer movement frequency; another at around $f_{2} \approx 16000 \mathrm{~Hz}$, which is believed to be the major frequency of the lower shear layer movement. 


\subsubsection{Spectral coherence}

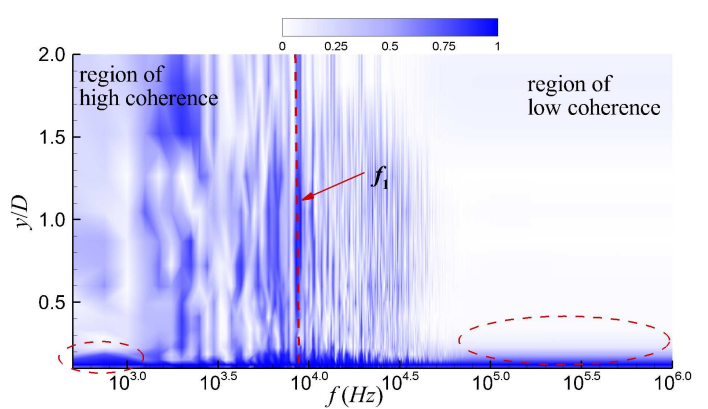

(a) $T^{\prime}$ coherence

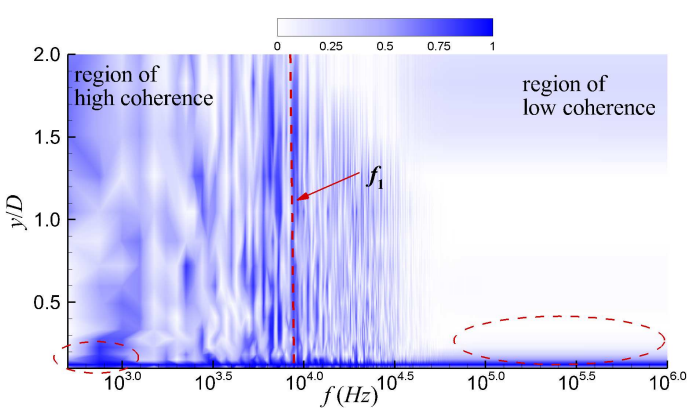

(b) $u^{\prime}$ coherence

Figure 26: Contours of coherence in the vertical direction in the wake of the 1st hole in case LT

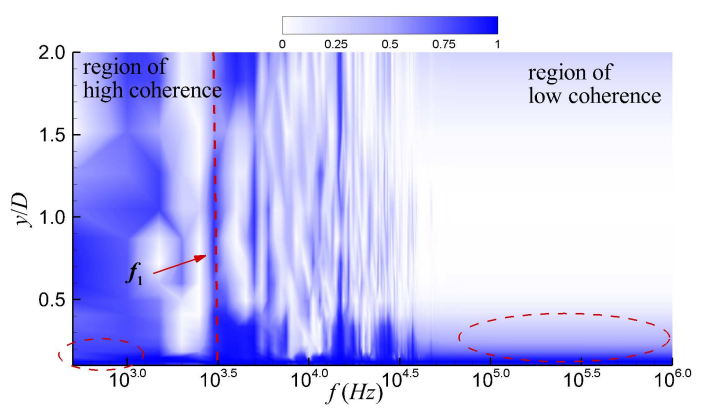

(a) $T^{\prime}$ coherence

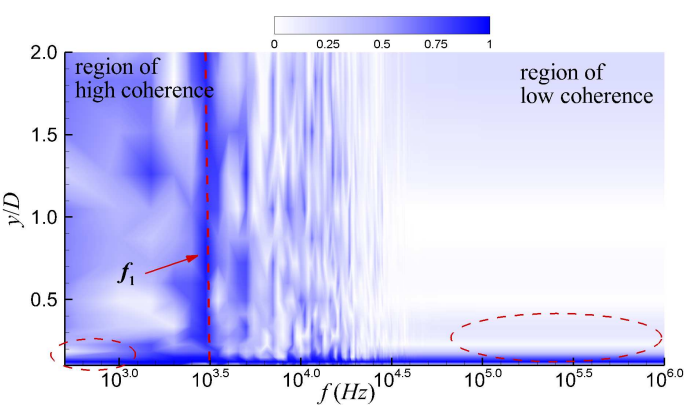

(b) $u^{\prime}$ coherence

Figure 27: Contours of coherence in the vertical direction in the wake of the 1st hole in case HT

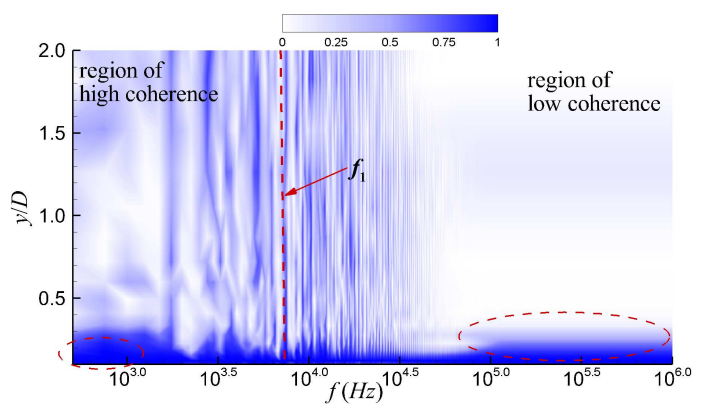

(a) $T^{\prime}$ coherence

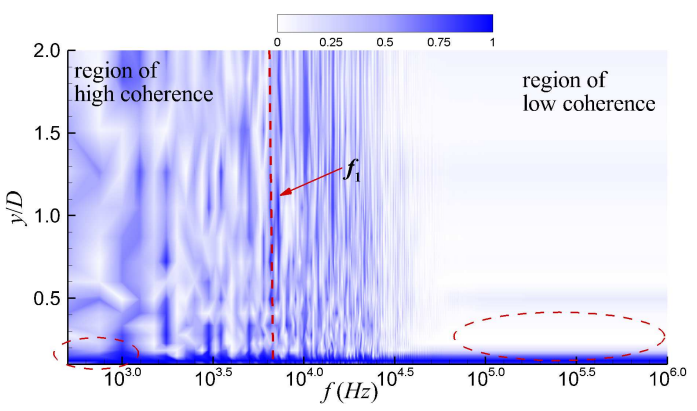

(b) $u^{\prime}$ coherence

Figure 28: Contours of coherence in the vertical direction in the wake of the 3rd hole in case LT

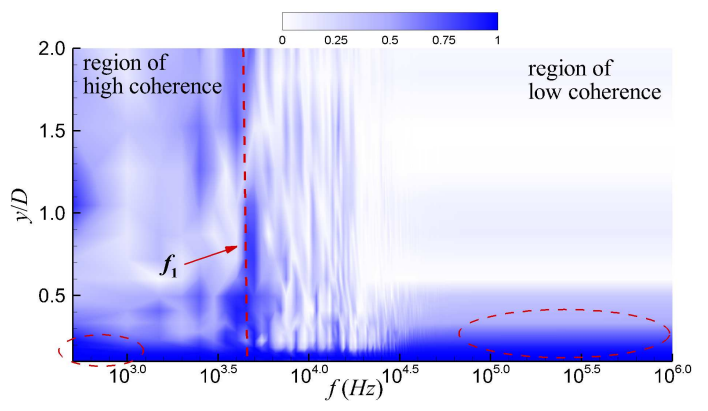

(a) $T^{\prime}$ coherence

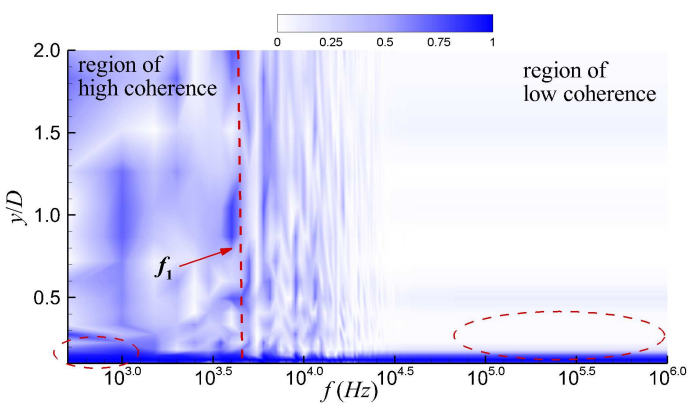

(b) $u^{\prime}$ coherence

Figure 29: Contours of coherence in the vertical direction in the wake of the 3rd hole in case HT 


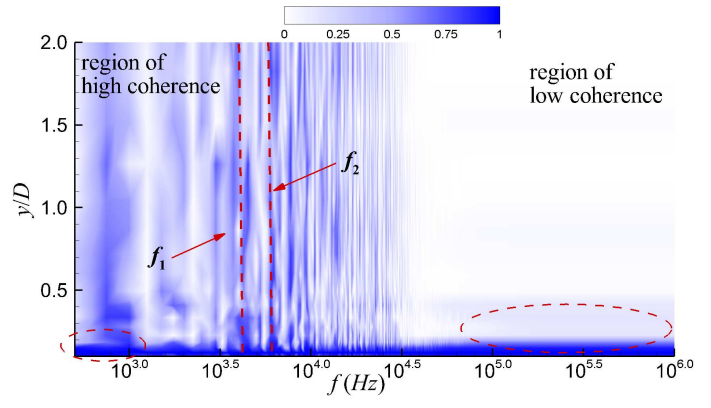

(a) $T^{\prime}$ coherence

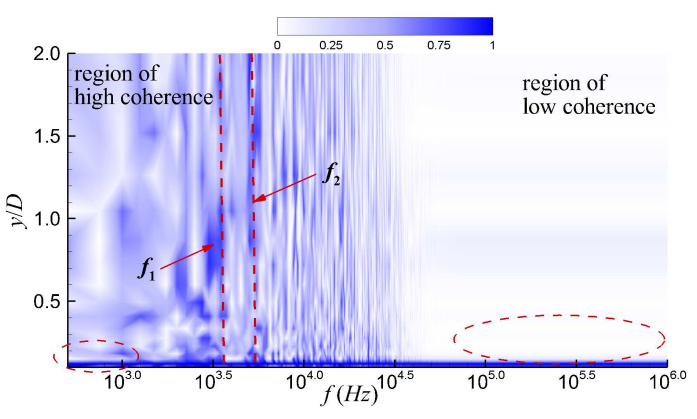

(b) $u^{\prime}$ coherence

Figure 30: Contours of coherence in the vertical direction in the wake of the 5th hole in case LT

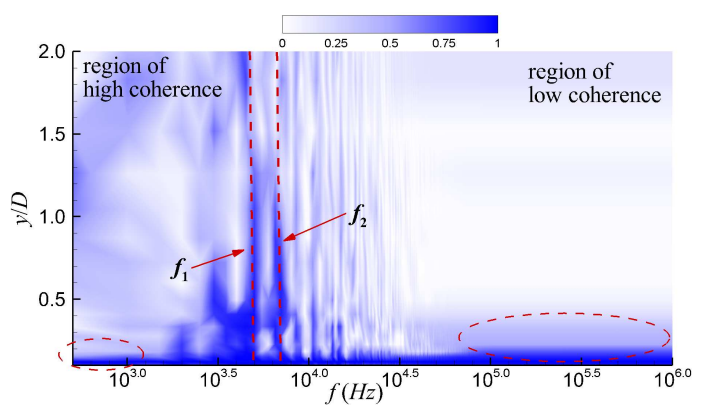

(a) $T^{\prime}$ coherence

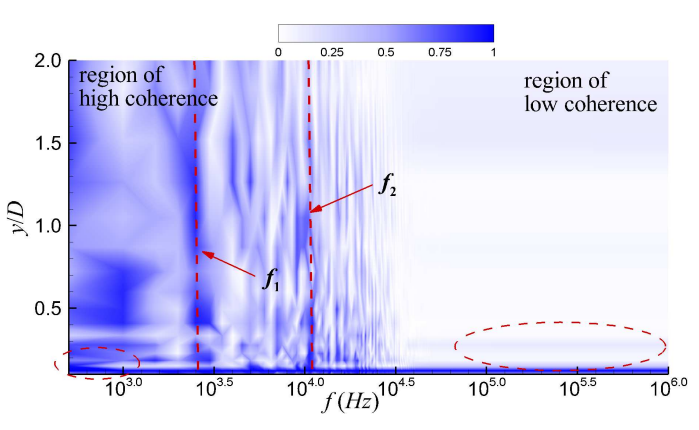

(b) $u^{\prime}$ coherence

Figure 31: Contours of coherence in the vertical direction in the wake of the 5th hole in case HT

Further study on the difference and similarity of the unsteady features in the wake of coolant jets are carried out by using the coherence function between two time-dependant signals. Following [41], the magnitude-squared coherence function between two temporal signals $a(t)$ and $b(t)$ is defined as,

$$
\mathscr{R}_{a b}^{2}(f)=\frac{\left|S_{a b}(f)\right|^{2}}{S_{a a}(f) S_{b b}(f)}
$$

where $S_{a b}(f)$ is the cross-spectral density of the signals $a(t)$ and $b(t), S_{a a}(f)$ and $S_{b b}(f)$ are the power spectral density functions of the corresponding signal, and the coherence function $\mathscr{R}_{a b}^{2}(f)$ varies between 0 and 1 . The two signals are said to be perfectly correlated at a certain frequency $f$ if the corresponding coherence function $\mathscr{R}_{a b}^{2}=1$. In this particular case, it suggests that turbulent flow structures with the same size occurs at both the position of $a$ and $b$. On the contrary, the two signals are uncorrelated at a certain frequency $f$ while the corresponding coherence function $\mathscr{R}_{a b}^{2}=0$. The spectral coherence analysis is a common tool in signal post-processing and has been in used various studies such as noise and vibration transmission [42], vibro-acoustics detection [43], electrical impedance measurements [44] etc.

In this section, the coherence of temperature and velocity fluctuations are studied between the nearwall position and the corresponding vertical positions as Figure 23 shows. The contours in Figures 26. 28 and 30 illustrate the coherence at different rows in case LT, while Figures 27, 29] and 31 presents the coherence in case HT. The contours stop at a vertical position of $y=2 D$ since the region of interest is within the coolant film region $(y<2 D)$, and especially at the coolant jet upper shear layer $(y \approx 1 D)$ and near-wall region. 
Overall, similarities can be found between the coherence contours of case LT and case HT. It is clear that both the temperature and velocity signals in the near-wall region have a number of frequency mods that are coherent with the upper part of the flow in the low frequency part $(f<30000 \mathrm{~Hz})$, although the coherent frequencies of case LT and case HT are slightly different. However, the coherence in the high frequency range $(f>30000 H z)$ is limited only in the near-wall region $(y<0.3 D)$, as the coherence above $0.3 D$ is close to 0 .

There's a clear difference between the coherence contours of case LT and case HT outside the nearwall region $(y>0.3 D)$. The coherent frequencies in case LT is more discrete and concentrate to a large number of narrow bands. Meanwhile, the temperature and velocity signals in case HT have a strong coherence at fewer but wider frequency bands. This is mainly due to that the high intensity inflow turbulence having a much stronger disturbance on the coolant jets and thus changing the flow mixing between the coolant jets and mainstream.

Comparing the coherence contours at the 1st row with the 3rd and 5th rows in case LT, it is clearly seen that both the temperature and velocity signals are highly coherent around $8000 \mathrm{~Hz}$ in the 1 st row, especially in the top part $(y>0.3 D)$. However, the coherence at such frequency reduces in the 3rd and 5th rows, while the lower frequencies are more coherent. Similar trend is observed in case HT with a high coherence at around $4000 \mathrm{~Hz}$ in the 1st row. The 3rd row also shows some coherence at such frequency despite a drop in the strength. However, the $u^{\prime}$ coherence in the 5 th row presents a strong coherence at a dropped frequency around $3000 \mathrm{~Hz}$, and a second coherent frequency at $10000 \mathrm{~Hz}$. Such difference in the coherence between the 1st and downstream rows is because the previous observation that the upstream coolant jet forms a coolant film and influences the downstream coolant jet behaviour. The differences in the 3rd row between case LT and case HT are due to the early breakdown of the 1st coolant jet in case HT.

Comparing the coherence maps of $T^{\prime}$ and $u^{\prime}$, it is found that the temperature signals are coherent at similar frequencies of the velocity signals in the upper region $(y>0.3 D)$ of the 1 st and $3 r d$ rows. It should be noted that the coherent frequencies of $T^{\prime}$ is different from that of $u^{\prime}$ in the 5 th row for both case LT and case HT. The coherent frequencies of $T^{\prime}$ stay similar to the previous rows, while that of $u^{\prime}$ moves to a lower frequency and a new high coherent frequency appears. This is due to the interaction between the local and upstream coolant jets introducing a different behaviour of the velocity shear layer. Meanwhile, the behaviour of the temperature shear layer is less influenced compared to the velocity field. In the near-wall region $(y<0.3 D)$, the coherence contours of the velocity signals are similar at different situations, while that of the temperature signals show significant changes both in case LT and case HT. In case LT, the area of coherence is larger in the 3rd row at both the low (around $1000 \mathrm{~Hz}$ ) and high frequency regions (above $10^{5} \mathrm{~Hz}$ ). In case HT, the area of coherence in the low frequency (around $1000 \mathrm{~Hz}$ ) is reducing from the 1 st row to the 5 th row, while the 3rd row shows the largest area in the high frequency region (above $10^{5} \mathrm{~Hz}$ ).

It is suggested by the coherence studies that the low frequency temperature fluctuation is closely related to the unsteady coolant jet motions. Meanwhile, the high frequency temperature fluctuation in the near-wall region is less correlated with the structures in the coolant jet away from the wall, but 
more owing to the local small turbulent structures in the near-wall region. Another finding from the coherence analysis is that the undisturbed near-wall structures are mostly coherent with the coolant shear structures in the high frequency region. However, the those high frequency coherence reduces when the coolant jet is affected by the inflow turbulence or upstream turbulent wake, and the coherent part moves to the low frequency region. This again illustrates the effects of incoming turbulent structures on the performance of coolant jet, which is difficult to distinguish from the measured spanwise-averaged ACE profiles.

\section{Conclusion}

The performance of combustor representative effusion cooling arrays with cylindrical coolant holes has been studied both numerically and experimentally. The ACE has been measured by the Pressure Sensitive Paint technique at blowing ratios from 1.81 to 4.08, with low and high levels of upstream fluctuation at $T u \approx 5 \%$ (LT) and $T u \approx 20 \%$ (HT) respectively. The high upstream fluctuation cases are used to study the cooling performance under realistic combustor inflow, while the low upstream fluctuation cases are used as a comparison to study the effects of different upstream fluctuation conditions. It is found from the measurements that increasing the amount of coolant from $B R=1.81$ to $B R=4.08$ improves the cooling performance by up to $4 \%$ in HT, but this is not effective (no more than $2 \%$ increase) in LT. It is also found that HT leads to a wider spreading of the coolant in the spanwise direction, with shorter streamwise coverage after the first few rows compared to LT. These effects also affected the spanwiseaveraged ACE from low to high BRs, but is more evident at low BRs (4\% difference in ACE) than high BRs (2\% difference).

More detailed study of the flow physics and cooling performance has been carried out using a hybrid LES method. Two simulations have been conducted at a blowing ratio of 1.81 with 5\% (LT) and $20 \%$ (HT) inflow fluctuation levels. The numerical method seems to perform better in the HT case, which is an interesting point to be investigated in the future study. It is found that increasing the level of upstream fluctuation results in enhanced mixing between the coolant and the hot mainstream, especially in the near-wall region. This result in a much earlier breakup of the coolant jets in the first few rows of the array in the HT case, which is shown by the study of coolant film thickness study where a sudden drop in the thickness is observed at $x / D=12$. The coolan jet centreline is also affected, the first coolant jet centreline in the HT case at $x=11 D$ is $0.8 D$ lower compared to LT case. This confirms the observation in the measured ACE contours, and demonstrated the effects quantitatively. Moreover, analysis suggests these effects become weaker when the coolant jets overlap and form a coolant film. The study of spectral frequency shows clear differences between case LT and case HT in the dominant frequencies, and the coherent frequencies between near-wall structures and coolant jet structures. It is also suggested that the low frequency fluctuation of the temperature field in the near-wall region is related to the large scale flow structure from the coolant jet, but the high frequency temperature fluctuation is purely determined by the small flow structures in the near-wall region.

Our numerical study confirms the findings from the measurement and reveals some detailed interactions between the turbulent inflow and coolant jets. This leads to potential further work on time- 
dependent measurements. Another extension of the current work could be on the performance of different coolant hole geometries, while the numerical simulation could benefit from lengthscale studies for even more complete pictures.

\section{Acknowledgments}

The experimental part of this work is funded by Rolls Royce and the Technology Strategy Board. The authors would also like to acknowledge the use of the "Hydra" High Performance Computing System at Loughborough University. Financial and technical support from the Department of Aeronautical and Automotive Engineering and the Rolls-Royce UTC at Loughborough University made this work possible.

\section{Nomenclature}

\begin{tabular}{|c|c|c|c|}
\hline$\delta_{i j}$ & Kronecer delta & $M$ & Mach number \\
\hline$\gamma$ & central-upwind blending parameter & $N u$ & Nusselt number, $N u=h L / k_{\text {fluid }}$ \\
\hline$\kappa$ & thermal conductivity & $p$ & static pressure of the fluid \\
\hline$\kappa_{T}$ & turbulent conductivity & $\operatorname{Pr}$ & Prandtl number \\
\hline$\tau$ & stress tensor, $=\left[\tau_{i j}\right]$ & $P r_{T}$ & turbulent Prandtl number \\
\hline $\mathbf{F}$ & inviscid flux vector, $=\left[\mathbf{F}_{i}\right]$ & $R$ & gas constant \\
\hline G & viscous flux vector, $=\left[\mathbf{G}_{i}\right]$ & $R e$ & Reynolds number, $R e=\rho U L / \mu$ \\
\hline Q & vector of primitive variables & $S_{i j}$ & strain-rate tensor \\
\hline $\mathbf{q}$ & heat flux vector, $=\left[q_{i}\right]$ & $S t$ & Strouhal number, $S t=f L / U$ \\
\hline $\mathbf{u}$ & $\begin{array}{l}\text { Cartesian components of velocity, }=\left[u_{i}\right]= \\
{[u, v, w]^{T}}\end{array}$ & $T$ & temperature \\
\hline W & vector of conservative variables & $t$ & physical time \\
\hline $\mathbf{x}$ & Cartesian coordinates, $=\left[x_{i}\right]=[x, y, z]^{T}$ & $t^{*}$ & non-dimensional time unit \\
\hline$\mu$ & viscosity & $T_{c}$ & coolant flow temperature \\
\hline$\mu_{T}$ & RANS eddy viscosity & $T_{\infty}$ & mainstream temperature \\
\hline$\mu_{\text {sgs }}$ & sub-grid scale eddy viscosity & $T u$ & turbulence intensity \\
\hline$\rho$ & fluid density & $U_{c}$ & coolant flow bulk velocity at exit \\
\hline$B i$ & Biot number, $B i=h L / k_{\text {solid }}$ & $U_{\infty}$ & mainstream velocity \\
\hline$c_{p}$ & specific heat & $\mathrm{ACE}$ & adiabatic coolant-film effectiveness \\
\hline$D$ & diameter of the coolant hole & $\mathrm{BR}$ & blowing ratio, $\rho_{c} U_{c} / \rho_{\infty} U_{\infty}$ \\
\hline$E$ & total energy per unit mass & $\mathrm{DR}$ & density ratio, $\rho_{c} / \rho_{\infty}$ \\
\hline$L_{T}$ & turbulent integral lengthscale & MR & momentum ratio, $\rho_{c} U_{c}^{2} / \rho_{\infty} U_{\infty}^{2}$ \\
\hline
\end{tabular}




\section{References}

[1] B. Facchini, L. Tarchi, L. Toni, A. Ceccherini, Adiabatic and overall effectiveness measurements of an effusion cooling array for turbine endwall application, J. Turbomach. 132 (4) (2010) 041008041008-11.

[2] A. Ceccerini, B. Facchini, L. Tarchi, D. Toni, D. Coutandin, Combined effect of slot injection, effusion array and dilution hole on the cooling performance of a real combustor liner, in: ASME Turbo Expo, American Society of Mechanical Engineers, 2009, pp. GT2009-60047.

[3] J. Dittmar, A. Schulz, S. Wittig, Assessment of various film-cooling configurations including shaped and compound angle holes based on large-scale experiments, J. Turbomach. 125 (1) (2003) 57-64.

[4] D. Martin, Effects of high intensity, large-scale free-stream turbulence on combustor effusion cooling, Ph.D. thesis, Loughborough University, Loughborough, UK (2013).

[5] J. Sargison, S. Guo, M. Oldfield, A. Rawlinson, The variation of heat transfer coefficient, adiabatic effectiveness and aerodynamic loss with film cooling hole shape, Annals of the New York Academy of Sciences 934 (1) (2001) 361-368.

[6] T. V. Jones, Theory for the use of foreign gas in simulating film cooling, Int. J. Heat Fluid Fl. 20 (3) (1999) 349-354.

[7] D. A. Gebbie, M. F. Reeder, C. Tyler, V. Fonov, J. Crafton, PSP-based experimental investigation of a blended wing body aircraft, in: 23rd AIAA Applied Aerodynamics Conference, American Institute of Aeronautics and Astronautics, 2005, pp. AIAA2005-4719.

[8] K. R. Navarra, Development of the pressure-sensitive-paint technique for advanced turbomachinery applications, M.Sc. thesis, Virginia Polytechnic Institute and State University, Blacksburg, VA, USA (1997).

[9] P. Steiner, Application of the pressure sensitive paint technique to the turbomachinery environment, Ph.D. thesis, Swiss Federal Institute of Technology (1997).

[10] J. Ahn, S. Mhetras, J.-C. Han, Film-cooling effectiveness on a gas turbine blade tip using pressure sensitive paint, Journal of Heat Transfer 127 (5) (2005) 521-530.

[11] L. J. Zhang, R. S. Jaiswal, Turbine nozzle endwall film cooling study using pressure-sensitive paint, Journal of Turbomachinery 123 (4) (2001) 730-738.

[12] G. Wang, G. Ledezma, J. DeLancey, A. Wang, Experimental study of effusion cooling with pressure sensitive paint (psp), Journal of Engineering for Gas Turbines and Power 139. doi:10.1115/1.4034943

[13] S. Park, E. Y. Jung, S. Kim, H.-S. Sohn, H. H. Cho, Enhancement of film cooling effectiveness using backward injection holes, International Journal of Thermal Sciences 110. doi:10.1016/j.ijthermalsci.2016.08.001. 
[14] B. E. Launder, J. York, Discrete-hole cooling in the presence of free stream turbulence and strong favourable pressure gradient, Int. J. Heat Mass Tran. 17 (11) (1974) 1403-1409.

[15] K. Kadotani, R. J. Goldstein, Effect of mainstream variables on jets issuing from a row of inclined round holes, J. Eng. P. 101 (2) (1979) 298-304.

[16] A. Kohli, D. G. Bogard, Effects of very high free-stream turbulence on the jet-mainstream interaction in a film cooling flow, J. Turbomach. 120 (4) (1998) 785-790.

[17] J. Ziefle, L. Kleiser, Numerical investigation of a film-cooling flow structure: Effect of crossflow turbulence, J. Turbomach. 135 (4) (2013) 041001:1-12.

[18] B. Wurm, A. Schulz, H.-J. Bauer, M. Gerendas, Impact of swirl flow on the cooling performance of an effusion cooled combustor liner, J. Eng. Gas Turb. Power 134 (12).

[19] A. Andreini, R. Becchi, B. Facchini, L. Mazzei, A. Picchi, F. Turrini, Adiabatic effectiveness and flow field measurements in a realistic effusion cooled lean burn combustor, J. Eng. Gas Turb. Power 138 (3) (2016) 031506.

[20] D. Martin, S. Thorpe, Experiments on combustor effusion cooling under conditions of very high free-stream turbulence, in: ASME Turbo Expo, 2012.

[21] A. K. Sinha, D. G. Bogard, M. E. Crawford, Film-cooling effectiveness downstream of a single row of holes with variable density ratio, J. Turbomach. 113 (3) (1991) 442-449.

[22] D. R. Pedersen, E. R. G. Eckert, R. J. Goldstein, Film cooling with large density differences between the mainstream and the secondary fluid measured by the heat-mass transfer analogy, J. Heat Transf. 99 (1977) 620-627.

[23] J. Krawciw, D. Martin, P. Denman, Measurement and prediction of adiabatic film effectiveness of combustor representative effusion arrays, in: ASME Turbo Expo 2015: Turbine Technical Conference and Exposition, American Society of Mechanical Engineers, 2015, pp. V05BT12A035V05BT12A035.

[24] S. Kline, F. McClintock, Describing the uncertainties in single-sample experiments, Mech. Eng. 75.

[25] T. Liu, J. Sullivan, Pressure and temperature sensitive paints, Springer, 2005.

[26] A. Schulz, Infrared thermography as applied to film cooling of gas turbine components, Measurement Science and Technology 11 (7) (2000) 948.

[27] H. Xia, Dynamic grid detach-eddy simulation for synthetic jet flows, Ph.D. thesis, The University of Sheffield, Sheffield, UK (2005).

[28] H. Xia, P. G. Tucker, S. Eastwood, Large-eddy simulations of chevron jet flows with noise predictions, Int. J. Heat Fluid FL. 30 (6) (2009) 1067-1079. 
[29] H. Xia, Turbulent jet characteristics for axisymmetric and serrated nozzles, Comput. Fluids 110 (2014) 189-197.

[30] A. Sinha, G. Kristjan, H. Xia, T. Colonius, Parabolized stability analysis of jets from serrated nozzles, J. Fluid Mech. 789 (2016) 33-63.

[31] X. Chen, H. Xia, A hybrid LES-RANS study on square cylinder unsteady heat transfer, Int. J. Heat Mass Tran. 108 (2017) 1237-1254.

[32] X. Chen, H. Xia, Flow and surface heat transfer analysis of a square cylinder in turbulent cross-flow, Numerical Heat Transfer, Part A: Applications 75 (12) (2019) 795-823.

[33] F. R. Menter, Improved two-equation $k-\omega$ turbulence models for aerodynamic flows, Tech. Rep. TM 103975, NASA (1992).

[34] F. R. Menter, M. Kuntz, R. Langtry, Ten years of industrial experience with the SST turbulence model, in: K. Hanjalic, Y. Nagano, M. Tummers (Eds.), Turbulence, Heat Mass Transfer 4, Begell House Inc., 2003, pp. 625-632.

[35] N. Jarrin, S. Benhamadouche, D. Laurence, R. Prosser, A synthetic-eddy-method for generating inflow conditions for large-eddy simulations, Int. J. Heat Fluid Fl. 27 (4) (2006) 585-593.

[36] J. W. Kim, S. Haeri, An advanced synthetic eddy method for the computation of aerofoil-turbulence interaction noise, J. Comput. Phys. 287 (2015) 1-17.

[37] R. Poletto, T. Craft, A. Revell, A new divergence free synthetic eddy method for the reproduction of inlet flow conditions for LES, Flow Turbul. Combust. 91 (3) (2013) 519-539.

[38] A. Skillen, A. Revell, T. Craft, Accuracy and efficiency improvements in synthetic eddy methods., Int. J. Heat Fluid Fl. 62 (2016) 386-394.

[39] X. Chen, A hybrid LES-RANS approach for effusion cooling prediction, Ph.D. thesis, Loughborough University, Loughborough, UK (2018).

[40] H. Schlichting, K. Gersten, Boundary Layer Theory, Springer, 2016.

[41] K. Shin, J. Hammond, Fundamentals of signal processing for sound and vibration engineers, John Wiley \& Sons, 2008.

[42] A. G. González, J. Rodrıguez, X. Sagartzazu, A. Schumacher, I. Isasa, Multiple coherence method in time domain for the analysis of the transmission paths of noise and vibrations with non stationary signals, Proceedings of ISMA 2010.

[43] J. M. Muggleton, M. J. Brennan, Y. Gao, Determining the location of buried plastic water pipes from measurements of ground surface vibration, J. Appl. Geophysics 75 (1) (2011) $54-61$.

[44] R. A. Nazer, V. Cattin, P. Granjon, M. Montaru, M. Ranieri, Broadband identification of battery electrical impedance for HEVs, IEEE Transactions on Vehicular Technology 62 (7) (2013) 2896-2905. 